\title{
VARIATION OPERATORS FOR SEMIGROUPS AND RIESZ TRANSFORMS ON BMO IN THE SCHRÖDINGER SETTING
}

\author{
J.J. BETANCOR, J.C. FARIÑA, E. HARBOURE, AND L. RODRÍGUEZ-MESA
}

\begin{abstract}
In this paper we prove that the variation operators of the the heat semigroup and the truncations of Riesz transforms associated to the Schrödinger operator are bounded on a suitable $B M O$ type space.
\end{abstract}

\section{INTRODUCTION}

We consider the Schrödinger operator $\mathscr{L}$ defined by $\mathscr{L}=-\Delta+V$ on $\mathbb{R}^{n}, n \geq 3$. Here $V$ is a nonnegative and not identically zero function satisfying, for some $q>n / 2$, the following reverse Hölder inequality:

$\left(R H_{q}\right)$ There exists $C>0$ such that, for every ball $B \subset \mathbb{R}^{n}$,

$$
\left(\frac{1}{|B|} \int_{B} V(x)^{q} d x\right)^{1 / q} \leq C \frac{1}{|B|} \int_{B} V(x) d x .
$$

We write $V \in R H_{q}$ when $V$ verifies the property $\left(R H_{q}\right)$. Note that if $V$ is a nonnegative polynomial, then $V \in R H_{q}$ for every $1<q<\infty$. Also, if $V_{\alpha}(x)=|x|^{\alpha}, x \in \mathbb{R}^{n}, V_{\alpha}$ belongs to $R H_{q}$ provided that $\alpha q>-n$. Hence, $V_{\alpha} \in R H_{n / 2}$ when $\alpha>-2$, and $V_{\alpha} \in R H_{n}$ if $\alpha>-1$.

Harmonic analysis operators derived from Schrödinger differential operators (Riesz transforms, maximal operators associated with heat and Poisson semigroups for $\mathscr{L}$, Littlewood-Paley $g$-functions, fractional integrals,...) have been extensively studied in the last years. The papers of Zhong [36] and Shen [29] can be considered as starting points. In [29] and [36] Riesz transforms in the Schrödinger setting were studied on $L^{p}$-spaces. The behavior on $L^{p}$ of other operators related to $\mathscr{L}$ have been investigated in [5, [19, [25], 26] and [32, among others.

Dziubanski and Zienkiewicz introduced appropriate Hardy spaces associated with $\mathscr{L}$ (see [14], [15], and [16]). A function $f \in L^{1}\left(\mathbb{R}^{n}\right)$ is said to be in $H_{1}^{\mathscr{L}}\left(\mathbb{R}^{n}\right)$ if and only if $W_{*}^{\mathscr{L}}(f) \in L^{1}\left(\mathbb{R}^{n}\right)$, where

$$
W_{*}^{\mathscr{L}}(f)=\sup _{t>0}\left|W_{t}^{\mathscr{L}}(f)\right|,
$$

and $W^{\mathscr{L}}=\left\{W_{t}^{\mathscr{L}}\right\}_{t>0}$ denotes the heat semigroup generated by $-\mathscr{L}$.

The dual space of $H_{1}^{\mathscr{L}}\left(\mathbb{R}^{n}\right)$ was investigated in [13]. This dual space, denoted by $B M O^{\mathscr{L}}\left(\mathbb{R}^{n}\right)$, was characterized as the natural space of bounded mean oscillation functions in this setting. More precisely, a function $f \in L_{\text {loc }}^{1}\left(\mathbb{R}^{n}\right)$ is said to be in $B M O^{\mathscr{L}}\left(\mathbb{R}^{n}\right)$ provided that there exists $C>0$ such that the following two properties are satisfied:

(i) For every $x \in \mathbb{R}^{n}$ and $r>0$,

$$
\frac{1}{|B(x, r)|} \int_{B(x, r)}\left|f(y)-f_{B(x, r)}\right| d y \leq C,
$$

where, as usual, $f_{B(x, r)}=\frac{1}{|B(x, r)|} \int_{B(x, r)} f(y) d y$, and $|B(x, r)|$ denotes the Lebesgue measure of the ball $B(x, r)$; and

(ii) For every $x \in \mathbb{R}^{n}$ and $r \geq \gamma(x)$,

$$
\frac{1}{|B(x, r)|} \int_{B(x, r)}|f(y)| d y \leq C .
$$

Here, for any $x$, the critical radius $\gamma(x)$ is defined by

$$
\gamma(x)=\sup \left\{r>0: r^{2-n} \int_{B(x, r)} V(y) d y \leq 1\right\} .
$$

This paper is partially supported by MTM2007/65609. 
Since $V$ is not identically zero and $V \in R H_{q}$ with $q>n / 2$, it follows that $0<\gamma(x)<\infty$. The norm $\|f\|_{B M O^{\mathscr{L}}\left(\mathbb{R}^{n}\right)}$ of $f \in B M O^{\mathscr{L}}\left(\mathbb{R}^{n}\right)$ is defined by

$$
\|f\|_{B M O^{\mathscr{L}}\left(\mathbb{R}^{n}\right)}=\inf \{C>0: \text { (i) and (ii) hold }\} .
$$

In [13] the behavior of certain maximal operators, Littlewood-Paley $g$ functions and fractional integrals on $B M O^{\mathscr{L}}\left(\mathbb{R}^{n}\right)$ were studied. Also, the $B M O^{\mathscr{L}}\left(\mathbb{R}^{n}\right)$-boundedness of the Riesz transforms has been analyzed in [4, [12] and [35].

Suppose that $\left\{T_{t}\right\}_{t>0}$ is a family of operators defined for functions in $L^{p}\left(\mathbb{R}^{n}\right), 1 \leq p<\infty$. If $\rho>2$, the $\rho$-variation operator associated with $\left\{T_{t}\right\}_{t>0}, V_{\rho}\left(T_{t}\right)$, is defined by

$$
V_{\rho}\left(T_{t}\right)(f)(x)=\sup _{\left\{t_{j}\right\}_{j \in \mathbb{N} \downarrow} 0}\left(\sum_{j=1}^{\infty}\left|T_{t_{j}}(f)(x)-T_{t_{j+1}}(f)(x)\right|^{\rho}\right)^{1 / \rho},
$$

where the supremum is taken over all the real decreasing sequences $\left\{t_{j}\right\}_{j \in \mathbb{N}}$ that converge to zero. The operator $V_{\rho}\left(T_{t}\right)$ is related to the convergence of $T_{t}$, as $t \rightarrow 0^{+}$, and it estimates the fluctuations near the origin of the family $\left\{T_{t}\right\}_{t>0}$.

We consider the linear space $E_{\rho}$ that consists of all those real functions $F$ defined on $(0, \infty)$ such that

$$
\|F\|_{E_{\rho}}=\sup _{\left\{t_{j}\right\}_{j \in \mathbb{N} \downarrow 0}}\left(\sum_{j=1}^{\infty}\left|F\left(t_{j}\right)-F\left(t_{j+1}\right)\right|^{\rho}\right)^{\frac{1}{\rho}}<\infty,
$$

where the supremum is taken over all the decreasing real sequence $\left\{t_{j}\right\}_{j=1}^{\infty}$ that converge to zero. $\left(E_{\rho},\|\|_{E_{\rho}}\right)$ is a seminorm on $E_{\rho}$. The variation operator $V_{\rho}\left(T_{t}\right)$ can be rewritten in the following way

$$
V_{\rho}\left(T_{t}\right)(f)(x)=\left\|T_{t}(f)(x)\right\|_{E_{\rho}} .
$$

The variation operator $V_{\rho}$ was introduced in the ergodic context by Bourgain [6] (see also Jones et al. [21]). In the last years many authors have investigated the variation operator associated to semigroups of operators and singular integrals ([7, [8], [10, [11, [18, [20, [23] and [24]). Recently, Oberlin, Seeger, Tao, Thiele and Wright [27] have analyzed the variation norm related to Carleson Theorem.

In a previous paper (see [2]) the authors studied the $L^{p}$-boundedness properties of the variation operators for the heat semigroup $\left\{W_{t}^{\mathscr{L}}\right\}_{t>0}$ and the family of truncated Riesz transforms $\left\{R_{\ell}^{\mathscr{L}, \varepsilon}\right\}_{\varepsilon>0}, \ell=1, \cdots, n$, in the Schrödinger context. Here our aim is to study the behavior of the variation operators $V_{\rho}\left(W_{t}^{\mathscr{L}}\right)$ and $V_{\rho}\left(R_{\ell}^{\mathscr{L}, \varepsilon}\right)$ acting on functions in $B M O^{\mathscr{L}}\left(\mathbb{R}^{n}\right)$. Previously, we shall analyze the variation operators $V_{\rho}\left(W_{t}\right)$ and $V_{\rho}\left(R_{\ell}^{\varepsilon}\right)$ over the classical $B M O\left(\mathbb{R}^{n}\right)$, where $\left\{W_{t}\right\}_{t>0}$ and $\left\{R_{\ell}^{\varepsilon}\right\}_{\varepsilon>0}, \ell=1,2, \cdots, n$, stand for the classical heat semigroup and the truncated Riesz transforms, respectively. As usual, by $B M O\left(\mathbb{R}^{n}\right)$ we denote the well known space of bounded mean oscillation functions in $\mathbb{R}^{n}$. We believe that these results in the classical setting are of independent interest.

This paper is organized as follows. In Section 2 we state our results and we also describe a quite general procedure that allows us to prove properties concerning the operators in the Schrödinger setting by using the corresponding results for the classical (Euclidean) context. The proofs of the theorems are presented in Sections 3 (classical setting) and 4 (Schrödinger context).

\section{Comment: no se si es muy cierto esto de que describimos el procedimiento gen- eral..}

Throughout this paper we denote by $C$ a positive constant that may change from one line to another.

\section{Main Results}

In this section we present the main results of the paper, stated as Theorems $2.2,2.4,2.6$ and 2.8 below.

As it is well known the heat semigroup $\left\{W_{t}\right\}_{t>0}$ generated by $-\Delta$ is defined, for every $f \in$ $L^{p}\left(\mathbb{R}^{n}\right), 1 \leq p \leq \infty$, by

$$
W_{t}(f)(x)=(4 \pi t)^{-n / 2} \int_{\mathbb{R}^{n}} e^{-|x-y|^{2} / 4 t} f(y) d y, \quad x \in \mathbb{R}^{n} .
$$


The $L^{p}$-boundedness properties of the variation operator $V_{\rho}\left(W_{t}\right), \rho>2$, were studied in [22, Theorem 3.3] and [11, Theorem 1.1]. We provide here the precise statement.

Theorem 2.1. ([22, Theorem 3.3] and [11, Theorem 1.1]) If $\rho>2$, the variation operator $V_{\rho}\left(W_{t}\right)$ is bounded from $L^{p}\left(\mathbb{R}^{n}\right)$ into itself, for every $1<p<\infty$, and from $L^{1}\left(\mathbb{R}^{n}\right)$ into $L^{1, \infty}\left(\mathbb{R}^{n}\right)$.

In [11] it was shown that the variation operator $V_{\rho}\left(W_{t}\right)$ is not bounded on $L^{\infty}\left(\mathbb{R}^{n}\right)$. In fact, in [11, Section 5], the authors give an example of a function $f \in L^{\infty}\left(\mathbb{R}^{n}\right)$ ) such that $V_{\rho}\left(W_{t}\right)(f)(x)=\infty$, a.e. $x \in \mathbb{R}$, for every $\rho>2$. As it is well known $L^{\infty}\left(\mathbb{R}^{n}\right)$ is a subset of the space $B M O\left(\mathbb{R}^{n}\right)$ of bounded mean oscillation functions. In the next result we take care of the behavior of $V_{\rho}\left(W_{t}\right)$ on $B M O\left(\mathbb{R}^{n}\right)$.

Theorem 2.2. Let $\rho>2$. Then, if $f \in B M O\left(\mathbb{R}^{n}\right)$ and $V_{\rho}\left(W_{t}\right) f(x)<\infty$, a.e. $x \in \mathbb{R}^{n}$, $V_{\rho}\left(W_{t}\right) f \in B M O\left(\mathbb{R}^{n}\right)$ and $\left\|V_{\rho}\left(W_{t}\right) f\right\|_{B M O\left(\mathbb{R}^{n}\right)} \leq C\|f\|_{B M O\left(\mathbb{R}^{n}\right)}$.

For every $\ell=1, \cdots, n$, the Riesz transform $R_{\ell}(f)$ of $f \in L^{p}\left(\mathbb{R}^{n}\right), 1 \leq p<\infty$, is given by

$$
R_{\ell}(f)(x)=\lim _{\varepsilon \rightarrow 0^{+}} c_{n} \int_{|x-y|>\varepsilon} \frac{x_{\ell}-y_{\ell}}{|x-y|^{n+1}} f(y) d y, \text { a.e. } x \in \mathbb{R}^{n},
$$

where $c_{n}=\Gamma((n+1) / 2) / \pi^{(n+1) / 2}$.

Regarding the variation operator for $R_{\ell}, \ell=1, \ldots, n$, their $L^{p}$-boundedness was investigated in [7] and 8]. We reproduce here their precise statement.

Theorem 2.3. (77, Theorem 1.2] and [8, Theorem A and Corollary 1.4]). Let $\ell=1, \ldots, n$. If $\rho>2$, the variation operator $V_{\rho}\left(R_{\ell}^{\varepsilon}\right)$ is bounded from $L^{p}\left(\mathbb{R}^{n}\right)$ into itself, for every $1<p<\infty$, and from $L^{1}\left(\mathbb{R}^{n}\right)$ into $L^{1, \infty}\left(\mathbb{R}^{n}\right)$.

By using transference methods Gillespie and Torrea ([18, Theorem B]) obtained dimension free $L^{p}\left(\mathbb{R}^{n},|x|^{\alpha} d x\right)$ norm inequalities, for every $1<p<\infty$ and $-1<\alpha<p-1$, for variation operators of the Riesz transform $R_{\ell}, \ell=1, \ldots, n$. Using the idea developed in the proof of [18, Lemma 1.4], we are able to analyze the behavior of the operators $V_{\rho}\left(R_{\ell}^{\varepsilon}\right)$ on the space $B M O\left(\mathbb{R}^{n}\right)$.

First notice that for $\ell=1, \cdots, n, f \in B M O\left(\mathbb{R}^{n}\right)$ and $\varepsilon>0$, the integral $\int_{|x-y|>\varepsilon} f(y) \frac{y_{\ell}-x_{\ell}}{|y-x|^{n+1}} d y$ may be non-convergent. Indeed, for instance, the function $f(x)=\frac{1}{\log (x+2)} \chi_{(0, \infty)}(x), x \in \mathbb{R}$, belongs to $L^{\infty}(\mathbb{R}) \subset B M O(\mathbb{R})$ but the limit $\lim _{N \rightarrow \infty} \int_{\varepsilon<|x-y|<N} \frac{f(y)}{x-y} d y$ does not exist, for any $x \in \mathbb{R}$ and $\varepsilon>0$. However, it is clear that, for every $0<\varepsilon<\eta, \int_{\varepsilon<|x-y|<\eta} \frac{|f(y)|}{|x-y|^{n}} d y<\infty$ for any $x \in \mathbb{R}^{n}$. Therefore, in this situation, the operators $V_{\rho}\left(R_{\ell}^{\varepsilon}\right)$ can be defined on $B M O\left(\mathbb{R}^{n}\right)$ in the obvious way, that is, by replacing $R_{\ell}^{\varepsilon_{j}}(f)(x)-R_{\ell}^{\varepsilon_{j+1}}(f)(x)$ by $\int_{\varepsilon_{j+1}<|x-y|<\varepsilon_{j}} f(y) \frac{y_{\ell}-x_{\ell}}{|y-x|^{n+1}} d y$, $\ell=1, \cdots, n$ and $j \in \mathbb{N}$. Let us mention that in [10, Theorem B] it was proved that for $f \in L^{\infty}(\mathbb{R})$ and $\rho>2$ then either $V_{\rho}\left(H^{\varepsilon}\right)(f)(x)=\infty$, a.e. $x \in \mathbb{R}$, or $V_{\rho}\left(H^{\varepsilon}\right)(f)(x)<\infty$, a.e. $x \in \mathbb{R}$, where $H$ denotes the Riesz transform on $\mathbb{R}$, that is, the Hilbert transform. Moreover, as it can be seen in [10, Section 1], if $f(x)=\operatorname{sgn}(x), x \in \mathbb{R}$, then $V_{\rho}\left(H^{\varepsilon}\right)(f)(x)=\infty$, a.e. $x \in \mathbb{R}$.

In the next result we establish the behavior of the variation operator $V_{\rho}\left(R_{\ell}^{\varepsilon}\right)$ for functions in $B M O\left(\mathbb{R}^{n}\right)$.

Theorem 2.4. Let $\ell=1, \ldots, n$ and $\rho>2$. Then, if $f \in B M O\left(\mathbb{R}^{n}\right)$ and $V_{\rho}\left(R_{\ell}^{\varepsilon}\right)(f)(x)<\infty$, a.e. $x \in \mathbb{R}^{n}, V_{\rho}\left(R_{\ell}^{\varepsilon}\right)(f) \in B M O\left(\mathbb{R}^{n}\right)$ and $\left\|V_{\rho}\left(R_{\ell}^{\varepsilon}\right) f\right\|_{B M O\left(\mathbb{R}^{n}\right)} \leq C\|f\|_{B M O\left(\mathbb{R}^{n}\right)}$.

We turn now to the Schrödinger operator setting. Let us denote by $\left\{W_{t}^{\mathscr{L}}\right\}_{t>0}$ the heat semigroup associated with $\mathscr{L}$. For every $t>0$, they can be written in integral form

$$
W_{t}^{\mathscr{L}}(f)(x)=\int_{\mathbb{R}^{n}} W_{t}^{\mathscr{L}}(x, y) f(y) d y, f \in L^{2}\left(\mathbb{R}^{n}\right) .
$$

Even though we do not have an explicit formula for the kernels $W_{t}^{\mathscr{L}}(x, y)$, many properties are known and can be encountered, for instance, in [13].

$L^{p}$-boundedness properties of the variation operator $V_{\rho}\left(W_{t}^{\mathscr{L}}\right)$ were studied in $[2]$. We reproduce here the exact result.

Theorem 2.5. ([2, Theorem 1.1]). Let $V \in R H_{q}$ where $q>n / 2$ and let $\rho>2$. Then, the variation operator $V_{\rho}\left(W_{t}^{\mathscr{L}}\right)$ is bounded from $L^{p}\left(\mathbb{R}^{n}\right)$ into itself, for every $1<p<\infty$, and from $L^{1}\left(\mathbb{R}^{n}\right)$ into $L^{1, \infty}\left(\mathbb{R}^{n}\right)$.

Our next result shows the behavior of the variation operator $V_{\rho}\left(W_{t}^{\mathscr{L}}\right)$ on $\mathrm{BMO}^{\mathscr{L}}\left(\mathbb{R}^{n}\right)$. 
Theorem 2.6. Let $V \in R H_{q}$ where $q>n / 2$ and let $\rho>2$. Then, the variation operator $V_{\rho}\left(W_{t}^{\mathscr{L}}\right)$ is bounded from $\mathrm{BMO}^{\mathscr{L}}\left(\mathbb{R}^{n}\right)$ into itself.

Let $\ell=1, \cdots, n$. The Riesz transform $R_{\ell}^{\mathscr{L}}$ is defined by

$$
R_{\ell}^{\mathscr{L}}(f)=\frac{\partial}{\partial x_{\ell}} \mathscr{L}^{-1 / 2} f, \quad f \in C_{c}^{\infty}\left(\mathbb{R}^{n}\right),
$$

where $C_{c}^{\infty}\left(\mathbb{R}^{n}\right)$ denotes the space of smooth functions with compact support in $\mathbb{R}^{n}$. Here, the negative square root $\mathscr{L}^{-1 / 2}$ of $\mathscr{L}$ is defined in terms of the heat semigroup by

$$
\mathscr{L}^{-1 / 2}(f)(x)=\frac{1}{\sqrt{\pi}} \int_{0}^{\infty} W_{t}^{\mathscr{L}}(f)(x) t^{-1 / 2} d t .
$$

Fractional powers of the Schrödinger operator $\mathscr{L}$ have been studied in $[3]$.

In [2, Proposition 1.1] it was proved that $R_{\ell}^{\mathscr{L}}$ can be extended to $\left.L^{p}(\mathbb{R})^{n}\right)$ as the principal value operator

$$
R_{\ell}^{\mathscr{L}}(f)(x)=\lim _{\varepsilon \rightarrow 0^{+}} \int_{|x-y|>\varepsilon} R_{\ell}^{\mathscr{L}}(x, y) f(y) d y, \text { a.e. } x \in \mathbb{R}^{n},
$$

where

$$
R_{\ell}^{\mathscr{L}}(x, y)=-\frac{1}{2 \pi} \frac{\partial}{\partial x_{\ell}} \int_{\mathbb{R}}(-i \tau)^{-1 / 2} \Gamma(x, y, \tau) d \tau, x, y \in \mathbb{R}^{n}, x \neq y,
$$

Comment: No habria que explicar que es esta funcion $\Gamma(x, y, \tau)$ ?

provided that

(i) $1 \leq p<\infty$, and $V \in R H_{n}$

(ii) $1<p<p_{0}$, where $\frac{1}{p_{0}}=\frac{1}{q}-\frac{1}{n}$, and $V \in R H_{q}, n / 2 \leq q<n$.

Moreover, $R_{\ell}^{\mathscr{L}}$ is bounded from $L^{p}\left(\mathbb{R}^{n}\right)$ into itself when $1<p<\infty$ and from $L^{1}\left(\mathbb{R}^{n}\right)$ into $L^{1, \infty}\left(\mathbb{R}^{n}\right)$, provided that $V \in R H_{n}$. Also, $R_{\ell}^{\mathscr{L}}$ is bounded from $L^{p}\left(\mathbb{R}^{n}\right)$ into itself when $1<p<$ $p_{0}$ and $V \in R H_{q}$, with $n / 2 \leq q<n$ ([29, Theorem 0.5 and 0.8$\left.]\right)$.

The formal adjoint operator $\mathscr{R}_{\ell}^{\mathscr{L}}$ of $R_{\ell}^{\mathscr{L}}$ defined by

$$
\mathscr{R}_{\ell}^{\mathscr{L}}(f)(x)=\lim _{\varepsilon \rightarrow 0^{+}} \int_{|x-y|>\varepsilon} R_{\ell}^{\mathscr{L}}(y, x) f(y) d y, \text { a.e. } x \in \mathbb{R}^{n},
$$

is bounded from $L^{p}\left(\mathbb{R}^{n}\right)$ into itself when $p_{0}^{\prime}<p<\infty$ and $V \in R H_{q}$, with $n / 2 \leq q<n$, where as usual $p_{0}^{\prime}$ denotes the exponent conjugated to $p_{0}$. In the case that $V \in R H_{n}$ it is bounded for $1<p<\infty$ and it also maps $L^{1}\left(\mathbb{R}^{n}\right)$ into $L^{1, \infty}\left(\mathbb{R}^{n}\right)$.

By defining the truncated Riesz transforms $R_{\ell}^{\mathscr{L}, \varepsilon}, \varepsilon>0$, in the natural way, the $L^{p}$-boundedness properties for the variation operator $V_{\rho}\left(R_{\ell}^{\mathscr{L}, \varepsilon}\right)$ were established in [2].

Theorem 2.7. ([2, Theorem 1.2]. Let $\ell=1, \cdots, n$. Assume that $\rho>2$. Then, the variation operator $V_{\rho}\left(R_{\ell}^{\mathscr{L}, \varepsilon}\right)$ is bounded

(i) from $L^{p}\left(\mathbb{R}^{n}\right)$ into itself, $1<p<\infty$, and from $L^{1}\left(\mathbb{R}^{n}\right)$ into $L^{1, \infty}\left(\mathbb{R}^{n}\right)$, provided that $V \in R H_{n}$.

(ii) from $L^{p}\left(\mathbb{R}^{n}\right)$ into itself, when $1<p<p_{0}$, where $\frac{1}{p_{0}}=\frac{1}{q}-\frac{1}{n}$, and $V \in R H_{q}, n / 2 \leq q<n$.

If $f \in B M O^{\mathscr{L}}\left(\mathbb{R}^{n}\right)$ and $\ell=1, \cdots, n$, the limit in $\left[1\right.$ exists for a.e. $x \in \mathbb{R}^{n}$ (see [2]). Thus, the Riesz transforms $R_{\ell}^{\mathscr{L}}$ are defined by $\sqrt{1}$ in $B M O^{\mathscr{L}}\left(\mathbb{R}^{n}\right)$. As it was remarked earlier, the situation is quite different in the classical case.

In the next result we describe the behavior on $B M O^{\mathscr{L}}\left(\mathbb{R}^{n}\right)$ of the variation operators associated with the Riesz transforms $R_{\ell}^{\mathscr{L}}$ and their adjoints.

Theorem 2.8. Let $\rho>2$ and let $\ell=1, \cdots, n$. If $V \in R H_{q}$ where $q \geq n$, then the variation operator $V_{\rho}\left(R_{\ell}^{\mathscr{L}, \varepsilon}\right)$ is bounded from $B M O^{\mathscr{L}}\left(\mathbb{R}^{n}\right)$ into itself. Also, the variation operator $V_{\rho}\left(\mathscr{R}_{\ell}^{\mathscr{L}, \varepsilon}\right)$ is bounded from $B M O^{\mathscr{L}}\left(\mathbb{R}^{n}\right)$ into itself, provided that $V \in R H_{q}$ where $q>n / 2$.

Note that there is a remarkable difference between the results in the classical and in the Schrödinger settings. In the latter, the operators are defined in the whole $B M O^{\mathscr{L}}\left(\mathbb{R}^{n}\right)$, while in the classical case it is necessary to impose an additional "finiteness hypothesis". This fact was observed by the first time in 13 . 
In order to analyze operators in the Schrödinger context on $\mathrm{BMO}^{\mathscr{L}}\left(\mathbb{R}^{n}\right)$ we shall use some ideas developed in [13] and we will again exploit that the Schrödinger operator $\mathscr{L}=-\Delta+V$, where $V \in R H_{q}$, with $q>n / 2$, is actually a nice perturbation of the Laplacian operator $-\Delta$.

Throughout the proof of the results that we have just stated, the following properties will play an important role.

According to [13, Proposition 2.14] it is possible to choose a sequence $\left\{x_{k}\right\}_{k=1}^{\infty} \subset \mathbb{R}^{n}$, such that if $Q_{k}=B\left(x_{k}, \gamma\left(x_{k}\right)\right), k \in \mathbb{N}$, the following properties hold:

(i) $\cup_{k=1}^{\infty} Q_{k}=\mathbb{R}^{n}$;

(ii) For every $m \in \mathbb{N}$ there exist $C, \beta>0$ such that, for every $k \in \mathbb{N}$,

$$
\text { card }\left\{l \in \mathbb{N}: 2^{m} Q_{l} \cap 2^{m} Q_{k} \neq \varnothing\right\} \leq C 2^{m \beta} .
$$

Also, from [13, p. 346, after Lemma 9], for any operator $H$ and $f \in \mathrm{BMO}^{\mathscr{L}}\left(\mathbb{R}^{n}\right), H f \in$ $\mathrm{BMO}^{\mathscr{L}}\left(\mathbb{R}^{n}\right)$ provided that there exists a positive constant $C$ such that, for every $k \in \mathbb{N}$,

$\left(i_{k}\right) \frac{1}{\left|Q_{k}\right|} \int_{Q_{k}}|H(f)(x)| d x \leq C|| f \|_{\mathrm{BMO}^{\mathscr{L}}\left(\mathbb{R}^{n}\right)}$, and

$\left(i i_{k}\right) H f \in \operatorname{BMO}\left(Q_{k}^{*}\right)$ and $\|H f\|_{\mathrm{BMO}\left(Q_{k}^{*}\right)} \leq C\|f\|_{\mathrm{BMO}^{\mathscr{L}}\left(\mathbb{R}^{n}\right)}$. Here $\operatorname{BMO}\left(Q_{k}^{*}\right)$ denotes the usual BMO-space over $Q_{k}^{*}$.

Moreover, we have that

$$
\|H f\|_{B M O^{\mathscr{L}}\left(\mathbb{R}^{n}\right)} \leq M\|f\|_{B M O^{\mathscr{L}}\left(\mathbb{R}^{n}\right)},
$$

where the constant $M>0$ depends only on the constant $C$.

Note that if the property $\left(i_{k}\right)$ above holds for every $k \in \mathbb{N}$, then $|H(f)(x)|<\infty$ for almost every $x \in \mathbb{R}^{n}$. This fact is quite different from what happens in the classical Euclidean case (see Theorem 2.2 and Theorem 2.4 [1]).

\section{Proof of Theorems 2.2 and 2.4}

In this section we show our results about the behavior of the variation operator for the classical heat semigroup and Riesz transforms on $B M O\left(\mathbb{R}^{n}\right)$.

3.1. Proof of Theorem 2.2. Let $\rho>2$. Assume that $f \in B M O\left(\mathbb{R}^{n}\right)$ and $V_{\rho}\left(W_{t}\right)(f)(x)<\infty$, a.e. $x \in \mathbb{R}^{n}$. Let $B=B\left(x_{0}, r_{0}\right)$, with $x_{0} \in \mathbb{R}^{n}$ and $r_{0}>0$. We write

$$
f=\left(f-f_{B}\right) \chi_{B^{*}}+\left(f-f_{B}\right) \chi_{\left(B^{*}\right)^{c}}+f_{B}=f_{1}+f_{2}+f_{3} .
$$

Note that this type of decomposition allows us to see that $W_{t}(|f|)<\infty$. According to Theorem 2.1. we have

$$
\int_{\mathbb{R}^{n}}\left|V_{\rho}\left(W_{t}\right)\left(f_{1}\right)(x)\right|^{2} d x \leq C \int_{B^{*}}\left|f(x)-f_{B}\right|^{2} d x \leq C|B|\|f\|_{B M O\left(\mathbb{R}^{n}\right)}^{2} .
$$

In particular this means that $V_{\rho}\left(W_{t}\right)\left(f_{1}\right)(x)<\infty$, a.e. $x \in \mathbb{R}^{n}$. Moreover, since $\left\{W_{t}\right\}_{t \geq 0}$ is Markovian, $V_{\rho}\left(W_{t}\right)\left(f_{3}\right)=0$. Then, using the hypothesis, we may choose $x_{1} \in B\left(x_{0}, r_{0}\right)$ such that $V_{\rho}\left(W_{t}\right)\left(f_{2}\right)\left(x_{1}\right)<\infty$.

If $E_{\rho}$ denotes the space introduced in Section 1, we can write

$$
\begin{aligned}
\frac{1}{|B|} \int_{B} \mid V_{\rho}\left(W_{t}\right)(f)(x) & -V_{\rho}\left(W_{t}\right)\left(f_{2}\right)\left(x_{1}\right) \mid d x \\
& =\frac{1}{|B|} \int_{B}\left|\left\|W_{t}(f)(x)\right\|_{E_{\rho}}-\left\|W_{t}\left(f_{2}\right)\left(x_{1}\right)\right\|_{E_{\rho}}\right| d x \\
& \leq \frac{1}{|B|} \int_{B}\left\|W_{t}(f)(x)-W_{t}\left(f_{2}\right)\left(x_{1}\right)\right\|_{E_{\rho}} d x \\
& =\frac{1}{|B|} \int_{B}\left\|W_{t}\left(f_{1}\right)(x)+W_{t}\left(f_{2}\right)(x)-W_{t}\left(f_{2}\right)\left(x_{1}\right)\right\|_{E_{\rho}} d x \\
\leq & \frac{1}{|B|} \int_{B}\left\|W_{t}\left(f_{1}\right)(x)\right\|_{E_{\rho}} d x+\frac{1}{|B|} \int_{B}\left\|W_{t}\left(f_{2}\right)(x)-W_{t}\left(f_{2}\right)\left(x_{1}\right)\right\|_{E_{\rho}} d x .
\end{aligned}
$$

Therefore, according to 22 we get

$$
\frac{1}{|B|} \int_{B}\left\|W_{t}\left(f_{1}\right)(x)\right\|_{E_{\rho}} d x \leq\left(\frac{1}{|B|} \int_{B}\left|V_{\rho}\left(W_{t}\right)\left(f_{1}\right)(x)\right|^{2} d x\right)^{\frac{1}{2}} \leq C\|f\|_{B M O\left(\mathbb{R}^{n}\right)} .
$$


Also, Minkowski inequality and [11, p. 91] lead to

(5) $\frac{1}{|B|} \int_{B}\left\|W_{t}\left(f_{2}\right)(x)-W_{t}\left(f_{2}\right)\left(x_{1}\right)\right\|_{E_{\rho}} d x$

$$
\begin{aligned}
& =\frac{1}{|B|} \int_{B}\left\|\int_{\mathbb{R}^{n}} W_{t}(x, y) f_{2}(y) d y-\int_{\mathbb{R}^{n}} W_{t}\left(x_{1}, y\right) f_{2}(y) d y\right\|_{E_{\rho}} d x \\
& \leq C \frac{1}{|B|} \int_{B} \int_{\mathbb{R}^{n}}\left\|W_{t}(x, y)-W_{t}\left(x_{1}, y\right)\right\|_{E_{\rho}}\left|f_{2}(y)\right| d y d x \\
& \leq \frac{C}{|B|} \int_{B} \int_{\left(B^{*}\right)} \frac{\left|x-x_{1}\right|}{|x-y|^{n+1}}\left|f(y)-f_{B}\right| d y d x \\
& \leq C \frac{r_{0}}{|B|} \int_{B} \int_{\left(B^{*}\right)^{c}} \frac{1}{\left(\left|y-x_{0}\right|-\left|x_{0}-x\right|\right)^{n+1}}\left|f(y)-f_{B}\right| d y d x \\
& \leq C \frac{r_{0}}{|B|} \int_{B} \sum_{k=1}^{\infty} \int_{2^{k} r_{0} \leq\left|y-x_{0}\right|<2^{k+1} r_{0}} \overline{\left(\left|y-x_{0}\right|-\left|x_{0}-x\right|\right)^{n+1}\left|f(y)-f_{B}\right| d y d x} \\
& \leq C \sum_{k=1}^{\infty} \frac{1}{2^{k}} \frac{1}{\left(2^{k} r_{0}\right)^{n}} \int_{\left|y-x_{0}\right|<2^{k+1} r_{0}}\left|f(y)-f_{B}\right| d y \leq C\|f\|_{B M O\left(\mathbb{R}^{n}\right) .}
\end{aligned}
$$

In the last inequality we have used the well known property (see [34, VIII, Proposition 3.2])

$$
\frac{1}{\left|2^{m} B\right|} \int_{2^{m} B}\left|f(y)-f_{B}\right| d y \leq C m\|f\|_{B M O\left(\mathbb{R}^{n}\right)}, \quad m \in \mathbb{N} .
$$

$>$ From (3), (4) and (5) we conclude that

$$
\frac{1}{|B|} \int_{B}\left|V_{\rho}\left(W_{t}\right)(f)(x)-V_{\rho}\left(W_{t}\right)\left(f_{2}\right)\left(x_{1}\right)\right| d x \leq C\|f\|_{B M O\left(\mathbb{R}^{n}\right)} .
$$

Thus, we have proved that $V_{\rho}\left(W_{t}\right)(f) \in B M O\left(\mathbb{R}^{n}\right)$.

Remark 3.1. After a careful reading of the proof of Theorem 2.2 we can deduce the following result that will be useful in the proof of Theorem 2.6.

Proposition 3.1. Let $\rho>2$ and $\mathscr{A}$ be a set of decreasing real sequences converging to zero. Assume that

$$
V_{\rho, \mathscr{A}}\left(W_{t}\right)(f)(x)=\sup _{\left\{t_{j}\right\}_{j \in \mathbb{N}} \in \mathscr{A}}\left(\sum_{j=1}^{\infty}\left|W_{t_{j}}(f)(x)-W_{t_{j+1}}(f)(x)\right|^{\rho}\right)^{1 / \rho}<\infty \text {, a.e. } x \in Q,
$$

where $Q$ is a ball in $\mathbb{R}^{n}$. Suppose that $f \in B M O\left(\mathbb{R}^{n}\right)$ and $B$ is a ball contained in $Q$. If we define $f_{2}=\left(f-f_{B}\right) \chi_{\left(B^{*}\right)^{c}}$ and choose $x_{1} \in B$ such that $V_{\rho, \mathscr{A}}\left(W_{t}\right)\left(f_{2}\right)\left(x_{1}\right)<\infty$, then there is a constant $C>0$ independent of $\mathscr{A}$ such that for any ball $B$

$$
\frac{1}{|B|} \int_{B}\left\|W_{t}(f)(x)-W_{t}\left(f_{2}\right)\left(x_{1}\right)\right\|_{E_{\rho, \mathscr{A}}} d x \leq C\|f\|_{B M O\left(\mathbb{R}^{n}\right)},
$$

where, for a function $h:(0, \infty) \mapsto \mathbb{C},\|h\|_{E_{\rho, \&}}$ means

$$
\|h\|_{E_{\rho, \mathscr{A}}}=\sup _{\left\{t_{j}\right\}_{j \in \mathbb{N} \in \mathscr{A}}}\left(\sum_{j=1}^{\infty}\left|h\left(t_{j}\right)-h\left(t_{j+1}\right)\right|^{\rho}\right)^{1 / \rho} .
$$

3.2. Proof of Theorem 2.4. Let $\rho>2$. Assume that $f \in B M O\left(\mathbb{R}^{n}\right)$ and that $V_{\rho}\left(R_{\ell}^{\varepsilon}\right)(f)(x)<$ $\infty$, a.e. $x \in \mathbb{R}^{n}$. To see that $V_{\rho}\left(R_{\ell}^{\varepsilon}\right)(f) \in B M O\left(\mathbb{R}^{n}\right)$ we extend to $\mathbb{R}^{n}$ the technique developed in the proof of [18, Lemma 1.4].

Let $B=B\left(x_{0}, r_{0}\right)$ be a ball in $\mathbb{R}^{n}$. As above, we decompose $f$ setting $f=f_{1}+f_{2}+f_{3}$, where $f_{1}=\left(f-f_{B}\right) \chi_{B^{* *}}, f_{2}=\left(f-f_{B}\right) \chi_{\left(B^{* *}\right)^{c}}$ and $f_{3}=f_{B}$. According to Theorem 2.3, we have

$$
\int_{\mathbb{R}^{n}}\left|V_{\rho}\left(R_{\ell}^{\varepsilon}\right)\left(f_{1}\right)(x)\right|^{2} d x \leq C \int_{B^{* *}}\left|f(x)-f_{B}\right|^{2} d x \leq C|B|\|f\|_{B M O\left(\mathbb{R}^{n}\right)}^{2} .
$$

Then, $V_{\rho}\left(R_{\ell}^{\varepsilon}\right)\left(f_{1}\right)(x)<\infty$, a.e. $x \in \mathbb{R}^{n}$. Moreover, $V_{\rho}\left(R_{\ell}^{\varepsilon}\right)\left(f_{3}\right)=0$. Then, we can choose $x_{1} \in B$ such that $V_{\rho}\left(R_{\ell}^{\varepsilon}\right)\left(f_{2}\right)\left(x_{1}\right)<\infty$. 
If $E_{\rho}$ denotes the space defined in Section 1, by $(6)$ we can write

(7) $\frac{1}{|B|} \int_{B}\left|V_{\rho}\left(R_{\ell}^{\varepsilon}\right)(f)(x)-V_{\rho}\left(R_{\ell}^{\varepsilon}\right)\left(f_{2}\right)\left(x_{1}\right)\right| d x$

$$
\begin{aligned}
& \leq \frac{1}{|B|} \int_{B}\left|\left\|R_{\ell}^{\varepsilon}(f)(x)\right\|_{E_{\rho}}-\left\|R_{\ell}^{\varepsilon}\left(f_{2}\right)\left(x_{1}\right)\right\|_{E_{\rho}}\right| d x \\
& \leq \frac{1}{|B|} \int_{B}\left\|R_{\ell}^{\varepsilon}(f)(x)-R_{\ell}^{\varepsilon}\left(f_{2}\right)\left(x_{1}\right)\right\|_{E_{\rho}} d x \\
& \leq \frac{1}{|B|} \int_{B}\left\|R_{\ell}^{\varepsilon}\left(f_{1}\right)(x)\right\|_{E_{\rho}} d x+\frac{1}{|B|} \int_{B}\left\|R_{\ell}^{\varepsilon}\left(f_{2}\right)(x)-R_{\ell}^{\varepsilon}\left(f_{2}\right)\left(x_{1}\right)\right\|_{E_{\rho}} d x \\
& \leq C\|f\|_{B M O\left(\mathbb{R}^{n}\right)}+\frac{1}{|B|} \int_{B}\left\|R_{\ell}^{\varepsilon}\left(f_{2}\right)(x)-R_{\ell}^{\varepsilon}\left(f_{2}\right)\left(x_{1}\right)\right\|_{E_{\rho}} d x
\end{aligned}
$$

Denoting $R_{\ell}(z)=c_{n} \frac{z_{\ell}}{|z|^{n+1}}, z=\left(z_{1}, \cdots, z_{n}\right) \in \mathbb{R}^{n} \backslash\{0\}$, we have that

$$
\left\|R_{\ell}^{\varepsilon}\left(f_{2}\right)(x)-R_{\ell}^{\varepsilon}\left(f_{2}\right)\left(x_{1}\right)\right\|_{E_{\rho}} \leq A_{1}(x)+A_{2}(x), \quad x \in B,
$$

where, for every $x \in B$,

$$
A_{1}(x)=\left\|\int_{|x-y|>\varepsilon}\left(R_{\ell}(x-y)-R_{\ell}\left(x_{1}-y\right)\right) f_{2}(y) d y\right\|_{E_{\rho}}
$$

and

$$
A_{2}(x)=\left\|\int_{\mathbb{R}^{n}}\left(\chi_{\{\varepsilon<|x-y|\}}(y)-\chi_{\left\{\varepsilon<\left|x_{1}-y\right|\right\}}(y)\right) R_{\ell}\left(x_{1}-y\right) f_{2}(y) d y\right\|_{E_{\rho}} .
$$

By using Minkowski inequality and well known properties of the function $R_{\ell}(z)$ we get

$$
\begin{aligned}
A_{1}(x) & \leq \int_{\mathbb{R}^{n}}\left|R_{\ell}(x-y)-R_{\ell}\left(x_{1}-y\right)\right|\left|f(y)-f_{B}\right| \chi_{\left(B^{* *}\right)^{c}}(y) d y \\
& \leq C \sum_{k=2}^{\infty} \int_{2^{k} r_{0} \leq\left|x_{0}-y\right| \leq 2^{k+1} r_{0}} \frac{\left|x-x_{1}\right|}{|x-y|^{n+1}}\left|f(y)-f_{B}\right| d y \\
& \leq C \sum_{k=1}^{\infty} \frac{1}{2^{k}} \frac{1}{\left(2^{k} r_{0}\right)^{k}} \int_{2^{k+1} B}\left|f(y)-f_{B}\right| d y \\
& \leq C\|f\|_{B M O\left(\mathbb{R}^{n}\right)}, \quad x \in B .
\end{aligned}
$$

In order to analyze $A_{2}$ we split, for every $j \in \mathbb{N}$, the integral there in four terms as follows. Let $\left\{\varepsilon_{j}\right\}_{j=1}^{\infty}$ be a real decreasing sequence that converges to zero. It follows that

$$
\begin{aligned}
& \int_{\mathbb{R}^{n}}\left|\chi_{\left\{\varepsilon_{j+1}<|x-y|<\varepsilon_{j}\right\}}(y)-\chi_{\left\{\varepsilon_{j+1}<\left|x_{1}-y\right|<\varepsilon_{j}\right\}}(y)\right|\left|R_{\ell}\left(x_{1}-y\right)\right|\left|f_{2}(y)\right| d y \\
& \leq C\left(\int_{\mathbb{R}^{n}} \chi_{\left\{\varepsilon_{j+1}<|x-y|<\varepsilon_{j+1}+r_{0}\right\}}(y) \chi_{\left\{\varepsilon_{j+1}<|x-y|<\varepsilon_{j}\right\}}(y)\left|\frac{1}{\left|x_{1}-y\right|^{n}}\right| f_{2}(y) \mid d y\right. \\
&+\int_{\mathbb{R}^{n}} \chi_{\left\{\varepsilon_{j}<\left|x_{1}-y\right|<\varepsilon_{j}+r_{0}\right\}}(y) \chi_{\left\{\varepsilon_{j+1}<|x-y|<\varepsilon_{j}\right\}}(y) \frac{1}{\left|x_{1}-y\right|^{n}}\left|f_{2}(y)\right| d y \\
&+\int_{\mathbb{R}^{n}} \chi_{\left\{\varepsilon_{j+1}<\left|x_{1}-y\right|<\varepsilon_{j+1}+r_{0}\right\}}(y) \chi_{\left\{\varepsilon_{j+1}<\left|x_{1}-y\right|<\varepsilon_{j}\right\}}(y) \frac{1}{\left|x_{1}-y\right|^{n}}\left|f_{2}(y)\right| d y \\
&\left.+\int_{\mathbb{R}^{n}} \chi_{\left\{\varepsilon_{j}<|x-y|<\varepsilon_{j}+r_{0}\right\}}(y) \chi_{\left\{\varepsilon_{j+1}<\left|x_{1}-y\right|<\varepsilon_{j}\right\}}(y) \frac{1}{\left|x_{1}-y\right|^{n}}\left|f_{2}(y)\right| d y\right) \\
&=C\left(A_{2,1}^{j}(x)+A_{2,2}^{j}(x)+A_{2,3}^{j}(x)+A_{2,4}^{j}(x)\right), \quad x \in B \text { and } j \in \mathbb{N} .
\end{aligned}
$$

Observe that if $x \in B$, then $A_{2, m}^{j}(x)=0$, when $m=1,3$ and $j \in \mathbb{N}$ and $r_{0} \geq \varepsilon_{j+1}$. Also, if $x \in B$, then $A_{2, m}^{j}(x)=0$, when $m=2,4$ and $j \in \mathbb{N}$ and $r_{0} \geq \varepsilon_{j}$. to

Let $j \in \mathbb{N}$. Since $\frac{4}{3}|x-y| \geq\left|x_{1}-y\right| \geq \frac{3}{4}|x-y|, y \notin B^{* *}$ and $x \in B$, Hölder inequality leads

$$
A_{2,1}^{j}(x) \leq C\left(\int_{\mathbb{R}^{n}} \chi_{\left\{\varepsilon_{j+1}<|x-y|<\varepsilon_{j}\right\}}(y) \frac{1}{|x-y|^{n s}}\left|f_{2}(y)\right|^{s} d y\right)^{\frac{1}{s}} v_{j+1}^{\frac{1}{s^{\prime}}}, \quad x \in B,
$$


and

$$
\begin{gathered}
A_{2,2}^{j}(x) \leq C\left(\int_{\mathbb{R}^{n}} \chi_{\left\{\max \left\{\varepsilon_{j+1}, \frac{3}{4} \varepsilon_{j}\right\}<|x-y|<\varepsilon_{j}\right\}}(y) \frac{1}{|x-y|^{n s}}\left|f_{2}(y)\right|^{s} d y\right)^{\frac{1}{s}} v_{j}^{\frac{1}{s^{\prime}}}, \quad x \in B, \\
A_{2,3}^{j}(x) \leq C\left(\int_{\mathbb{R}^{n}} \chi_{\left\{\varepsilon_{j+1}<\left|x_{1}-y\right|<\varepsilon_{j}\right\}}(y) \frac{1}{\left|x_{1}-y\right|^{n s}}\left|f_{2}(y)\right|^{s} d y\right)^{\frac{1}{s}} v_{j+1}^{\frac{1}{s^{\prime}}}, \quad x \in B,
\end{gathered}
$$

$$
A_{2,4}^{j}(x) \leq C\left(\int_{\mathbb{R}^{n}} \chi_{\left\{\max \left\{\varepsilon_{j+1}, \frac{3}{4} \varepsilon_{j}\right\}<\left|x_{1}-y\right|<\varepsilon_{j}\right\}}(y) \frac{1}{\left|x_{1}-y\right|^{n s}}\left|f_{2}(y)\right|^{s} d y\right)^{\frac{1}{s}} v_{j}^{\frac{1}{s^{\prime}}}, \quad x \in B .
$$

Here $1<s<\infty$ and $s^{\prime}=\frac{s}{s-1}$, and $v_{j}=\left(\varepsilon_{j}+r_{0}\right)^{n}-\varepsilon_{j}^{n}, j \in \mathbb{N}$. Note that $v_{j} \leq C \max \left\{r_{0}, \varepsilon_{j}\right\}^{n-1} r_{0}$, $j \in \mathbb{N}$, for a certain $C>0$.

We define the set $\mathscr{A}=\left\{j \in \mathbb{N}: r_{0}<\varepsilon_{j}\right\}$. We have that

Comment: tal vez la notacion no sea buena ya que se uso para otra cosa en la Proposicion 3.1

$$
\begin{aligned}
A_{2,1}^{j}(x) & \leq C \frac{v_{j+1}^{1 / s^{\prime}}}{\varepsilon_{j+1}^{(n-1) / s^{\prime}}}\left(\int_{\mathbb{R}^{n}} \chi_{\left\{\varepsilon_{j+1}<|x-y|<\varepsilon_{j}\right\}}(y) \frac{\left|f_{2}(y)\right|^{s}}{|x-y|^{n+s-1}} d y\right)^{1 / s} \\
& \leq C r_{0}^{1 / s^{\prime}}\left(\int_{\mathbb{R}^{n}} \chi_{\left\{\varepsilon_{j+1}<|x-y|<\varepsilon_{j}\right\}}(y) \frac{\left|f_{2}(y)\right|^{s}}{|x-y|^{n+s-1}} d y\right)^{1 / s},
\end{aligned}
$$

for every $x \in B$ and $j+1 \in \mathscr{A}$. In a similar way we can see that

$$
\begin{gathered}
A_{2,2}^{j}(x) \leq C r_{0}^{1 / s^{\prime}}\left(\int_{\mathbb{R}^{n}} \chi_{\left\{\varepsilon_{j+1}<|x-y|<\varepsilon_{j}\right\}}(y) \frac{\left|f_{2}(y)\right|^{s}}{|x-y|^{n+s-1}} d y\right)^{1 / s}, \quad x \in B \text { and } j \in \mathscr{A}, \\
A_{2,3}^{j}(x) \leq C r_{0}^{1 / s^{\prime}}\left(\int_{\mathbb{R}^{n}} \chi_{\left\{\varepsilon_{j+1}<\left|x_{1}-y\right|<\varepsilon_{j}\right\}}(y) \frac{\left|f_{2}(y)\right|^{s}}{\left|x_{1}-y\right|^{n+s-1}} d y\right)^{1 / s}, \quad x \in B \text { and } j+1 \in \mathscr{A},
\end{gathered}
$$

and

$$
A_{2,4}^{j}(x) \leq C r_{0}^{1 / s^{\prime}}\left(\int_{\mathbb{R}^{n}} \chi_{\left\{\varepsilon_{j+1}<\left|x_{1}-y\right|<\varepsilon_{j}\right\}}(y) \frac{\left|f_{2}(y)\right|^{s}}{\left|x_{1}-y\right|^{n+s-1}} d y\right)^{1 / s}, \quad x \in B \text { and } j \in \mathscr{A} .
$$

Hence, we get

$$
\begin{aligned}
\left(\sum_{j=1}^{\infty}\left|A_{2,1}^{j}(x)+A_{2,2}^{j}(x)\right|^{\rho}\right)^{1 / \rho} & \leq C\left(\sum_{j+1 \in \mathscr{A}}\left|A_{2,1}^{j}(x)\right|^{\rho}+\sum_{j \in \mathscr{A}}\left|A_{2,2}^{j}(x)\right|^{\rho}\right)^{1 / \rho} \\
& \leq C\left(\sum_{j \in \mathbb{N}}\left(\int_{\mathbb{R}^{n}} \chi_{\left\{\varepsilon_{j+1}<|x-y|<\varepsilon_{j}\right\}}(y) \frac{\left|f_{2}(y)\right|^{s}}{|x-y|^{n+s-1}} d y\right)^{\rho / s} r_{0}^{\rho / s^{\prime}}\right)^{1 / \rho} \\
& \leq C\left(\int_{\mathbb{R}^{n}} \frac{\left|f_{2}(y)\right|^{s}}{|x-y|^{n+s-1}} d y\right)^{1 / s} r_{0}^{1 / s^{\prime}} \\
& \leq C\left(\left(\sum_{k=1}^{\infty} \frac{1}{\left(2^{k} r_{0}\right)^{n}} \int_{\left|x_{0}-y\right|<2^{k+1} r_{0}}\left|f(y)-f_{B}\right|^{s} d y \frac{1}{2^{k(s-1)}}\right)^{1 / s}\right. \\
& \leq C\|f\|_{\mathrm{BMO}\left(\mathbb{R}^{n}\right)}, \quad x \in B .
\end{aligned}
$$

In a similar way we get

$$
\left(\sum_{j=1}^{\infty}\left|A_{2,3}^{j}(x)+A_{2,4}^{j}(x)\right|^{\rho}\right)^{1 / \rho} \leq C\|f\|_{\mathrm{BMO}\left(\mathbb{R}^{n}\right)}, \quad x \in B .
$$

From 10, 11 and 12 we infer that

$$
A_{2}(x) \leq C\|f\|_{\mathrm{BMO}\left(\mathbb{R}^{n}\right)}, \quad x \in B .
$$


Altogether (7), (8), (9) and (13) imply that

$$
\frac{1}{|B|} \int_{B}\left|V_{\rho}\left(R_{\ell}^{\varepsilon}\right)(f)(x)-V_{\rho}\left(R_{\ell}^{\varepsilon}\right)\left(f_{2}\right)\left(x_{1}\right)\right| d x \leq C\|f\|_{\mathrm{BMO}\left(\mathbb{R}^{n}\right)} .
$$

Thus, we have proved that $V_{\rho}\left(R_{\ell}^{\varepsilon}\right)(f) \in \operatorname{BMO}\left(\mathbb{R}^{n}\right)$.

By proceeding as in the above proof we can establish the following result that will be useful in the sequel.

Proposition 3.2. Let $a>0$ and $\ell=1,2, \ldots, n$. We define, for every $\varepsilon>0$ and $f \in L_{\mathrm{loc}}^{1}\left(\mathbb{R}^{n}\right)$,

$$
R_{\ell, a}^{\varepsilon}(f)(x)=\int_{\varepsilon<|x-y|<a} \frac{x_{\ell}-y_{\ell}}{|x-y|^{n+1}} d y .
$$

Then, if $\rho>2 V_{\rho}\left(R_{\ell, a}^{\varepsilon}\right)(f) \in \operatorname{BMO}\left(\mathbb{R}^{n}\right)$, provided that $f \in \operatorname{BMO}\left(\mathbb{R}^{n}\right)$.

Note that $V_{\rho}\left(R_{\ell, a}^{\varepsilon}\right)(f)(x)<\infty$, a.e. $x \in \mathbb{R}^{n}$, for every $f \in \operatorname{BMO}\left(\mathbb{R}^{n}\right)$ and $\ell=1, \cdots, n$. Indeed, let $f \in \operatorname{BMO}\left(\mathbb{R}^{n}\right)$ and $i=\ell, \cdots, n$. Suppose that $m \in \mathbb{N}$. Since $V_{\rho}\left(R_{\ell, a}^{\varepsilon}\right)(f) \leq V_{\rho}\left(R_{\ell}^{\varepsilon}\right)(f)$ and $f \in L_{\mathrm{loc}}^{2}\left(\mathbb{R}^{n}\right)$, according to Theorem 2.3 , we have that

$$
\begin{aligned}
\int_{B(0, m)} V_{\rho}\left(R_{\ell, a}^{\varepsilon}\right)(f)(x) d x & \leq \int_{B(0, m)} V_{\rho}\left(R_{\ell, a}^{\varepsilon}\right)\left(f \chi_{B(0, m+a)}\right)(x) d x \\
& \leq \int_{B(0, m)} V_{\rho}\left(R_{\ell}^{\varepsilon}\right)\left(f \chi_{B(0, m+a)}\right)(x) d x \\
& \leq \sqrt{m}\left(\int_{B(0, m)}\left(V_{\rho}\left(R_{\ell}^{\varepsilon}\right)\left(f \chi_{B(0, m+a)}\right)(x)\right)^{2} d x\right)^{1 / 2} \\
& \leq \sqrt{m}\left(\int_{B(0, m+a)}|f(x)|^{2}\right)^{1 / 2}<\infty .
\end{aligned}
$$

Comment: La primera desigualdad me parece igualdad. En las dos ultimas me parece que en lugar de $\sqrt{m}$ va $m^{n / 2}$.

Hence, $V_{\rho}\left(R_{\ell, a}^{\varepsilon}\right)(f)(x)<\infty$, a.e. $x \in B(0, m)$.

\section{Proof of Theorems 2.6 AND 2.8}

In this section we establish the boundedness in $B M O^{\mathscr{L}}\left(\mathbb{R}^{n}\right)$ of the variation operators for the heat semigroup and Riesz transforms in the Schrödinger setting.

4.1. Proof of Theorem 2.6. Let $\rho>0$. Assume that $f \in \mathrm{BMO}^{\mathscr{L}}\left(\mathbb{R}^{n}\right)$. Our goal is to show that $V_{\rho}\left(W_{t}^{\mathscr{L}}\right)(f)$ satisfies the properties $\left(i_{k}\right)$ and $\left(i i_{k}\right)$, for every $k \in \mathbb{N}$.

Fix $k \in \mathbb{N}$. We first prove $\left(i_{k}\right)$, that is, there exists $C>0$, independent of $k \in \mathbb{N}$, such that

$$
\frac{1}{\left|Q_{k}\right|} \int_{Q_{k}}\left|V_{\rho}\left(W_{t}^{\mathscr{L}}\right)(f)(x)\right| d x \leq C .
$$

We decompose $W_{t}^{\mathscr{L}}(f)$ as follows

$$
W_{t}^{\mathscr{L}}(f)(x)=H_{k, t}^{\mathscr{L}}(f)(x)+L_{k, t}^{\mathscr{L}}(f)(x), \quad x \in \mathbb{R}^{n} \text { and } t>0
$$

where

and

$$
H_{k, t}^{\mathscr{L}}(f)(x)=W_{t}^{\mathscr{L}}(f)(x) \chi_{\left\{t>\gamma\left(x_{k}\right)^{2}\right\}}(t), \quad x \in \mathbb{R}^{n} \text { and } t>0
$$

$$
L_{k, t}^{\mathscr{L}}(f)(x)=W_{t}^{\mathscr{L}}(f)(x) \chi_{\left\{0<t \leq \gamma\left(x_{k}\right)^{2}\right\}}(t), x \in \mathbb{R}^{n} \text { and } t>0 .
$$

Comment: Aqui arriba me parece mas claro decir que esta descomposicion se hace para $x \in Q_{k}$. Me suena raro hacerla para cualquier $x \in \mathbb{R}^{n}$. Lo mismo diria para la pagina 15. En caso de hacerlo asi en la desigualdad que sigue aqui abajo (it is clear that...)habria que poner la variable $x$ y agregar para $x \in Q_{k}$.

It is clear that

$$
V_{\rho}\left(W_{t}^{\mathscr{L}}\right)(f) \leq V_{\rho}\left(H_{k, t}^{\mathscr{L}}\right)(f)+V_{\rho}\left(L_{k, t}^{\mathscr{L}}\right)(f) .
$$


Let $\left\{t_{j}\right\}_{j \in \mathbb{N}}$ be a decreasing sequence that converges to zero. Suppose that $j_{k} \in \mathbb{N}$ is such that $t_{j_{k+1}} \leq \gamma\left(x_{k}\right)^{2}<t_{j_{k}}$. We can write

$$
\begin{aligned}
& \left(\sum_{j=1}^{\infty}\left|H_{k, t_{j}}^{\mathscr{L}}(f)(x)-H_{k, t_{j+1}}^{\mathscr{L}}(f)(x)\right|^{\rho}\right)^{1 / \rho} \\
\leq & \sum_{j=1}^{j_{k}-1}\left|W_{t_{j}}^{\mathscr{L}}(f)(x)-W_{t_{j+1}}^{\mathscr{L}}(f)(x)\right|+\left|W_{t_{j_{k}}}^{\mathscr{L}}(f)(x)\right| \\
\leq & \sum_{j=1}^{j_{k}-1}\left|\int_{t_{j+1}}^{t_{j}} \frac{\partial}{\partial t} W_{t}^{\mathscr{L}}(f)(x) d t\right|+\left|W_{t_{j_{k}}}^{\mathscr{L}}(f)(x)\right| \\
\leq & \int_{\gamma\left(x_{k}\right)^{2}}^{\infty} \int_{\mathbb{R}^{n}}\left|\frac{\partial}{\partial t} W_{t}^{\mathscr{L}}(x, y)\right||f(y)| d y d t+\sup _{t \geq \gamma\left(x_{k}\right)^{2}}\left|W_{t}^{\mathscr{L}}(f)(x)\right| \\
= & \Omega_{1, k}(f)(x)+\Omega_{2, k}(f)(x), \quad x \in \mathbb{R}^{n} .
\end{aligned}
$$

Hence

$$
V_{\rho}\left(H_{k, t}^{\mathscr{L}}\right)(f) \leq \Omega_{1, k}(f)+\Omega_{2, k}(f) .
$$

According to [13, Theorem 6], we get

$$
\frac{1}{\left|Q_{k}\right|} \int_{Q_{k}}\left|\Omega_{2, k}(f)(x)\right| d x \leq \frac{1}{\left|Q_{k}\right|} \int_{Q_{k}} \sup _{t>0}\left|W_{t}^{\mathscr{L}}(f)(x)\right| d x \leq C\|f\|_{B M O^{\mathscr{L}}\left(\mathbb{R}^{n}\right)} .
$$

By using [13, (2.11)], since $\gamma(x) \sim \gamma\left(x_{k}\right)$, when $x \in Q_{k}$, we obtain

$$
\begin{aligned}
& \Omega_{1, k}(f)(x) \leq C \int_{\gamma\left(x_{k}\right)^{2}}^{\infty} \int_{\mathbb{R}^{n}} \frac{|f(y)|}{t^{1+n / 2}} e^{-c|x-y|^{2} / t}\left(1+\frac{t}{\gamma\left(x_{k}\right)^{2}}\right)^{-1} d y d t \\
\leq & C \int_{\gamma\left(x_{k}\right)^{2}}^{\infty} \frac{1}{t^{1+n / 2}}\left(1+\frac{t}{\gamma\left(x_{k}\right)^{2}}\right)^{-1}\left(\int_{|x-y|<\sqrt{t}}+\sum_{m=0}^{\infty} \int_{\sqrt{t} 2^{m} \leq|x-y|<\sqrt{t} 2^{m+1}}\right)|f(y)|\left(1+\frac{|x-y|}{\sqrt{t}}\right)^{-1-n} d y d t \\
\leq & C \int_{\gamma\left(x_{k}\right)^{2}}^{\infty} \frac{1}{t^{1+n / 2}}\left(1+\frac{t}{\gamma\left(x_{k}\right)^{2}}\right)^{-1} \sum_{m=0}^{\infty} \frac{1}{2^{m(1+n)}} \int_{|x-y|<2^{m} \sqrt{t}}|f(y)| d y d t, \quad x \in Q_{k} .
\end{aligned}
$$

Moreover, by [13, Lemma 2], it follows

$$
\begin{aligned}
& \sum_{m=0}^{\infty} \frac{1}{2^{m(1+n)} t^{n / 2}} \int_{|x-y|<2^{m} \sqrt{t}}|f(y)| d y \\
\leq & \sum_{m \in \mathbb{N}, 2^{m} \sqrt{t} \leq \gamma(x)} \frac{1}{2^{m(1+n)} t^{n / 2}} \int_{|x-y|<2^{m} \sqrt{t}}|f(y)| d y+\sum_{m \in \mathbb{N}, 2^{m} \sqrt{t}>\gamma(x)} \frac{1}{2^{m(1+n) t^{n / 2}}} \int_{|x-y|<2^{m} \sqrt{t}}|f(y)| d y \\
\leq & C\|f\|_{B M O^{\mathscr{L}}\left(\mathbb{R}^{n}\right)}\left(\sum_{m \in \mathbb{N}, 2^{m} \sqrt{t} \leq \gamma(x)} \frac{1}{2^{m}}\left(1+\log \frac{\gamma(x)}{2^{m} \sqrt{t}}\right)+\sum_{m \in \mathbb{N}} \frac{1}{2^{m}}\right) \\
\leq & C\|f\|_{B M O^{\mathscr{L}}\left(\mathbb{R}^{n}\right)}\left(1+\log \frac{\sqrt{t}}{\gamma\left(x_{k}\right)}\right), t \geq \gamma\left(x_{k}\right)^{2} \text { and } x \in Q_{k} .
\end{aligned}
$$

Then,

$$
\begin{aligned}
\Omega_{1, k}(f)(x) & \leq C \int_{\gamma\left(x_{k}\right)^{2}}^{\infty}\left(1+\frac{t}{\gamma\left(x_{k}\right)^{2}}\right)^{-1}\left(1+\log \frac{\sqrt{t}}{\gamma\left(x_{k}\right)}\right) \frac{d t}{t}\|f\|_{B M O^{\mathscr{L}}\left(\mathbb{R}^{n}\right)} \\
& \leq C \int_{1}^{\infty}(1+\log (u)) \frac{d u}{u(1+u)}\|f\|_{B M O^{\mathscr{L}}\left(\mathbb{R}^{n}\right)} \\
& \leq C\|f\|_{B M O^{\mathscr{L}}\left(\mathbb{R}^{n}\right)}, x \in Q_{k} .
\end{aligned}
$$

Hence, we obtain

$$
\frac{1}{\left|Q_{k}\right|} \int_{Q_{k}} \Omega_{1, k}(f)(x) d x \leq C\|f\|_{B M O^{\mathscr{L}}\left(\mathbb{R}^{n}\right)} .
$$

By combining (16, 17) and (18) we get

$$
\frac{1}{\left|Q_{k}\right|} \int_{Q_{k}} V_{\rho}\left(H_{k, t}^{\mathscr{L}}\right)(f)(x) d x \leq C\|f\|_{B M O^{\mathscr{L}}\left(\mathbb{R}^{n}\right)}
$$


Here $C>0$ does not depend on $k$.

We now decompose $f$ as follows

$$
f=f \chi_{Q_{k}^{*}}+f \chi_{\left(Q_{k}^{*}\right)^{c}}=f_{1}+f_{2},
$$

where $Q_{k}^{*}=2 Q_{k}$.

It is clear that

$$
V_{\rho}\left(L_{k, t}^{\mathscr{L}}\right)(f) \leq V_{\rho}\left(L_{k, t}^{\mathscr{L}}\right)\left(f_{1}\right)+V_{\rho}\left(L_{k, t}^{\mathscr{L}}\right)\left(f_{2}\right) .
$$

By proceeding as above we get

$$
\begin{aligned}
V_{\rho}\left(L_{k, t}^{\mathscr{L}}\right)\left(f_{1}\right)(x) & \leq C \sup _{\left\{t_{j}\right\}_{j \in \mathbb{N} \downarrow 0}}\left(\sum_{j \in \mathbb{N}, t_{j} \leq \gamma\left(x_{k}\right)^{2}}\left|W_{t_{j}}^{\mathscr{L}}\left(f_{1}\right)(x)-W_{t_{j+1}}^{\mathscr{L}}\left(f_{1}\right)(x)\right|^{\rho}\right)^{1 / \rho} \\
& \left.+\sup _{0<t \leq \gamma\left(x_{k}\right)^{2}}\left|W_{t}^{\mathscr{L}}\left(f_{1}\right)(x)\right|\right) \\
& \leq C\left(V_{\rho}\left(W_{t}^{\mathscr{L}}\right)\left(f_{1}\right)(x)+W_{*}^{\mathscr{L}}\left(f_{1}\right)(x)\right), \quad x \in \mathbb{R}^{n} .
\end{aligned}
$$

Since $W_{*}^{\mathscr{L}}$ and $V_{\rho}\left(W_{t}^{\mathscr{L}}\right)$ are bounded operators from $L^{2}\left(\mathbb{R}^{n}\right)$ into itself (see Theorem 2.1 it follows that

$$
\begin{aligned}
\frac{1}{\left|Q_{k}\right|} \int_{Q_{k}} V_{\rho}\left(L_{k, t}^{\mathscr{L}}\right)\left(f_{1}\right)(x) d x & \leq\left(\frac{1}{\left|Q_{k}\right|} \int_{\mathbb{R}^{n}} V_{\rho}\left(L_{k, t}^{\mathscr{L}}\right)\left(f_{1}\right)(x)^{2} d x\right)^{1 / 2} \\
& \leq C\left(\frac{1}{\left|Q_{k}\right|} \int_{Q_{k}^{*}}|f(x)|^{2} d x\right)^{1 / 2} .
\end{aligned}
$$

Then, from [13, Corollary 3], we deduce that

$$
\frac{1}{\left|Q_{k}\right|} \int_{Q_{k}} V_{\rho}\left(L_{k, t}^{\mathscr{L}}\right)\left(f_{1}\right)(x) d x \leq C\|f\|_{B M O^{\mathscr{L}}\left(\mathbb{R}^{n}\right)} .
$$

On the other hand, we can write

$$
\begin{aligned}
V_{\rho}\left(L_{k, t}^{\mathscr{L}}\right)\left(f_{2}\right)(x) & \leq \int_{0}^{\gamma\left(x_{k}\right)^{2}} \int_{\left(Q_{k}^{*}\right)^{c}}\left|\frac{\partial}{\partial t} W_{t}^{\mathscr{L}}(x, y)\right||f(y)| d y d t+\sup _{0<t \leq \gamma\left(x_{k}\right)^{2}}\left|W_{t}^{\mathscr{L}}\left(f_{2}\right)(x)\right| \\
& =\Omega_{3, k}(f)(x)+\Omega_{4, k}(f)(x), \quad x \in \mathbb{R}^{n} .
\end{aligned}
$$

According to [13, (2.7)], for certain $C, c>0$, we get

$$
\begin{aligned}
\int_{0}^{\gamma\left(x_{k}\right)^{2}} \int_{\left(Q_{k}^{*}\right)^{c}}\left|\frac{\partial}{\partial t} W_{t}^{\mathscr{L}}(x, y)\right||f(y)| d y d t \\
\quad \leq \int_{|x-y|>\gamma\left(x_{k}\right)} \int_{0}^{\gamma\left(x_{k}\right)^{2}}\left|\frac{\partial}{\partial t} W_{t}^{\mathscr{L}}(x, y)\right| d t|f(y)| d y \\
\quad \leq C \int_{|x-y|>\gamma\left(x_{k}\right)}|f(y)| \int_{0}^{\gamma\left(x_{k}\right)^{2}} \frac{e^{-c|x-y|^{2} / t}}{t^{n / 2+1}} d t d y \\
\quad \leq C \int_{0}^{\gamma\left(x_{k}\right)^{2}} \frac{1}{t^{n / 2+1}} \sum_{j=0}^{\infty} \int_{2^{j} \gamma\left(x_{k}\right)<|x-y| \leq 2^{j+1} \gamma\left(x_{k}\right)}|f(y)|\left(\frac{t}{|x-y|^{2}}\right)^{(n+1) / 2} d y d t \\
\quad \leq C \int_{0}^{\gamma\left(x_{k}\right)^{2}} \frac{1}{\sqrt{t}} \sum_{j=0}^{\infty} \frac{1}{\left(2^{j} \gamma\left(x_{k}\right)\right)^{n+1}} \int_{|x-y| \leq 2^{j+1} \gamma\left(x_{k}\right)}|f(y)| d y d t \\
\leq C \sum_{j=0}^{\infty} \frac{1}{2^{j}\left(2^{j} \gamma\left(x_{k}\right)\right)^{n}} \int_{\left|x_{k}-y\right| \leq 2^{j+3} \gamma\left(x_{k}\right)}|f(y)| d y \leq C|| f \|_{\mathrm{BMO}^{\mathscr{L}}\left(\mathbb{R}^{n}\right)}, \quad x \in Q_{k} .
\end{aligned}
$$

Then, by 23 ,

$$
\frac{1}{\left|Q_{k}\right|} \int_{Q_{k}} \Omega_{3, k}(f)(x) d x \leq C\|f\|_{\mathrm{BMO}^{\mathscr{L}}\left(\mathbb{R}^{n}\right)} .
$$

Moreover, since $|f| \in \mathrm{BMO}^{\mathscr{L}}\left(\mathbb{R}^{n}\right)$, [13, Theorem 6] implies that

$$
\frac{1}{\left|Q_{k}\right|} \int_{Q_{k}} \Omega_{4, k}(f)(x) d x \leq \frac{1}{\left|Q_{k}\right|} \int_{Q_{k}} W_{*}^{\mathscr{L}}(|f|)(x) d x \leq C\|f\|_{\mathrm{BMO}^{\mathscr{L}}\left(\mathbb{R}^{n}\right)} .
$$


Hence, we conclude that

$$
\frac{1}{\left|Q_{k}\right|} \int_{Q_{k}} V_{\rho}\left(L_{k, t}^{\mathscr{L}}\right)\left(f_{2}\right)(x) d x \leq C\|f\|_{\mathrm{BMO}^{\mathscr{L}}\left(\mathbb{R}^{n}\right)} .
$$

By combining (21) and (24) we deduce

$$
\frac{1}{\left|Q_{k}\right|} \int_{Q_{k}} V_{\rho}\left(L_{k, t}^{\mathscr{L}}\right)(f)(x) d x \leq C\|f\|_{\mathrm{BMO}^{\mathscr{L}}\left(\mathbb{R}^{n}\right)} .
$$

Finally, 15, 19) and 25) imply that

$$
\frac{1}{\left|Q_{k}\right|} \int_{Q_{k}} V_{\rho}\left(W_{t}^{\mathscr{L}}\right)(f)(x) d x \leq C|| f \|_{\mathrm{BMO}^{\mathscr{L}}\left(\mathbb{R}^{n}\right)} .
$$

Note that $C>0$ does not depend on $k$.

Thus property $\left(i_{k}\right)$ is completely proved.

Now, we are going to prove assertion $\left(i i_{k}\right)$. Assume that $B=B\left(x_{0}, r_{0}\right) \subset Q_{k}^{*}$, with $x_{0} \in \mathbb{R}^{n}$ and $r_{0}>0$. Our purpose is to check that

$$
\frac{1}{|B|} \int_{B}\left|V_{\rho}\left(W_{t}^{\mathscr{L}}\right)(f)(x)-c_{B}\right| d x \leq C|| f \|_{\mathrm{BMO}^{\mathscr{L}}\left(\mathbb{R}^{n}\right)},
$$

for a certain constant $c_{B}$, and with $C>0$ independent of $k$ and $B$. To this end we decompose $W_{t}^{\mathscr{L}}$ as follows

$$
W_{t}^{\mathscr{L}}(f)=H_{k, t}^{\mathscr{L}}(f)+\left(L_{k, t}^{\mathscr{L}}(f)-L_{k, t}(f)\right)+L_{k, t}(f), \quad t>0,
$$

where $H_{k, t}^{\mathscr{L}}$ and $L_{k, t}^{\mathscr{L}}$ are defined as above, and

$$
L_{k, t}(f)=W_{t}(f) \chi_{\left\{0<t \leq \gamma\left(x_{k}\right)^{2}\right\}}(t), t>0 .
$$

Suppose that $c_{B}=\left\|h_{B}\right\|_{E_{\rho}}$ for some function $h$. Then, we can write

$$
\begin{aligned}
\left|V_{\rho}\left(W_{t}^{\mathscr{L}}\right)(f)(x)-c_{B}\right| & =\left|\left\|W_{t}^{\mathscr{L}}(f)(x)\right\|_{E_{\rho}}-\left\|h_{B}\right\|_{E_{\rho}}\right| \\
& \leq\left\|W_{t}^{\mathscr{L}}(f)(x)-h_{B}(t)\right\|_{E_{\rho}} \\
& \leq\left\|H_{k, t}^{\mathscr{L}}(f)(x)+\left(L_{k, t}^{\mathscr{L}}(f)(x)-L_{k, t}(f)(x)\right)+L_{k, t}(f)(x)-h_{B}(t)\right\|_{E_{\rho}} \\
& \leq\left\|H_{k, t}^{\mathscr{L}}(f)(x)\right\|_{E_{\rho}}+\left\|L_{k, t}^{\mathscr{L}}(f)(x)-L_{k, t}(f)(x)\right\|_{E_{\rho}}+\left\|L_{k, t}(f)(x)-h_{B}(t)\right\|_{E_{\rho}}
\end{aligned}
$$

Therefore, (26) will be proved if we are able to show the following three inequalities:

(A1) $\frac{1}{|B|} \int_{B}\left\|H_{k, t}^{\mathscr{L}}(f)(x)\right\|_{E_{\rho}} d x \leq C\|f\|_{\mathrm{BMO}^{\mathscr{L}}\left(\mathbb{R}^{n}\right)}$;

(A2) $\frac{1}{|B|} \int_{B}\left\|L_{k, t}^{\mathscr{L}}(f)(x)-L_{k, t}(f)(x)\right\|_{E_{\rho}} d x \leq C\|f\|_{\mathrm{BMO}^{\mathscr{L}}\left(\mathbb{R}^{n}\right)}$; and

(A3) $\frac{1}{|B|} \int_{B}\left\|L_{k, t}(f)(x)-h_{B}(t)\right\|_{E_{\rho}} d x \leq C|| f \|_{\mathrm{BMO}^{\mathscr{L}}\left(\mathbb{R}^{n}\right)}$,

for a certain function $h_{B}:(0, \infty) \mapsto \mathbb{C}$, and a constant $C>0$ independent of $k$ and $B$.

According to (16) we have

$$
V_{\rho}\left(H_{k, t}^{\mathscr{L}}\right)(f) \leq \Omega_{1, k}(f)+\Omega_{2, k}(f) .
$$

By proceeding as above we get

$$
\left|\Omega_{1, k}(f)(x)\right| \leq C|| f \|_{\mathrm{BMO}^{\mathscr{L}}\left(\mathbb{R}^{n}\right)}, \quad x \in Q_{k}^{*} .
$$

Moreover, by [13, (5.4)],

$$
\left|\Omega_{2, k}(f)(x)\right| \leq C|| f \|_{\mathrm{BMO}^{\mathscr{L}}\left(\mathbb{R}^{n}\right)}, \quad x \in Q_{k}^{*} .
$$

Then, from (28) and $(29),(A 1)$ holds.

To establish $(A 2)$ we firstly observe that

$$
\begin{aligned}
V_{\rho}\left(L_{k, t}^{\mathscr{L}}-L_{k, t}\right)(f)(x) & \leq \int_{0}^{\gamma\left(x_{k}\right)^{2}} \int_{\mathbb{R}^{n}}\left|\frac{\partial}{\partial t}\left(W_{t}^{\mathscr{L}}(x, y)-W_{t}(x, y)\right)\right||f(y)| d y d t \\
& +\sup _{0<t \leq \gamma\left(x_{k}\right)^{2}}\left|W_{t}^{\mathscr{L}}(f)(x)-W_{t}(f)(x)\right| \\
& =\Omega_{5, k}(f)(x)+\Omega_{6, k}(f)(x), \quad x \in \mathbb{R}^{n} .
\end{aligned}
$$

By [13, (5.5)] we get

$$
\Omega_{6, k}(f)(x) \leq C\|f\|_{\mathrm{BMO}^{\mathscr{L}}\left(\mathbb{R}^{n}\right)}, \quad x \in Q_{k}^{*} .
$$


The perturbation formula $([13,(5.25)])$ allows us to write

$$
\begin{aligned}
\frac{\partial}{\partial t}\left(W_{t}(x, y)-W_{t}^{\mathscr{L}}(x, y)\right) & =\int_{\mathbb{R}^{n}} V(z) W_{t / 2}^{\mathscr{L}}(x, z) W_{t / 2}(z, y) d z \\
& +\int_{0}^{t / 2} \int_{\mathbb{R}^{n}} V(z) \frac{\partial}{\partial t} W_{t-s}^{\mathscr{L}}(x, z) W_{s}(z, y) d z d s \\
& +\int_{t / 2}^{t} \int_{\mathbb{R}^{n}} V(z) W_{t-s}^{\mathscr{L}}(x, z) \frac{\partial}{\partial s} W_{s}(z, y) d z d s \\
& =\sum_{j=1}^{3} K_{j}(x, y, t), x, y \in \mathbb{R}^{n} \text { and } t>0 .
\end{aligned}
$$

According to [13, (2.3) and (2.8)], we get

$$
\begin{aligned}
\left|K_{1}(x, y, t)\right| & \leq C t^{-n} \int_{\mathbb{R}^{n}} V(z) e^{-\frac{|x-z|^{2}+|z-y|^{2}}{4 t}} d z \\
& \leq C t^{-n / 2} e^{-\frac{|x-y|^{2}}{16 t}} \int_{\mathbb{R}^{n}} V(z) t^{-n / 2} e^{-\frac{|x-z|^{2}}{8 t}} d z \\
& \leq C \gamma(x)^{-\delta} t^{-1+(\delta-n) / 2} e^{-\frac{|x-y|^{2}}{16 t}}, x, y \in \mathbb{R}^{n} \text { and } 0<t<\gamma\left(x_{k}\right)^{2} .
\end{aligned}
$$

Here and in the sequel $\delta$ represents a positive constant.

Moreover, by using [13, (2.7) and (2.8)], since $t / 2<t-s<t$ when $0<s<t / 2$, it follows that

$$
\begin{aligned}
\left|K_{2}(x, y, t)\right| & \leq C \int_{0}^{t / 2} \int_{\mathbb{R}^{n}} V(z) \frac{1}{(t-s)^{1+n / 2}} e^{-c \frac{|x-z|^{2}}{t-s}} \frac{1}{s^{n / 2}} e^{-\frac{|z-y|^{2}}{4 s}} d z d s \\
& \leq C \int_{0}^{t / 2} \int_{\mathbb{R}^{n}} V(z) \frac{1}{t^{1+n / 2}} e^{-c \frac{|x-z|^{2}}{t}} \frac{1}{s^{n / 2}} e^{-\frac{|z-y|^{2}}{4 s}} d z d s \\
& \leq C \frac{1}{t^{1+n / 2}} e^{-c \frac{|x-y|^{2}}{t}} \int_{0}^{t / 2} \int_{\mathbb{R}^{n}} V(z) \frac{1}{s^{n / 2}} e^{-c \frac{|z-y|^{2}}{s}} d z d s \\
& \leq C \frac{1}{t^{1+n / 2}} e^{-c \frac{|x-y|^{2}}{t}} \int_{0}^{t / 2} \frac{s^{-1+\delta / 2}}{\gamma(y)^{\delta}} d s \\
& \leq \gamma(y)^{-\delta} t^{-1+(\delta-n) / 2} e^{-c \frac{|x-y|^{2}}{t}}, x, y \in \mathbb{R}^{n} \text { and } 0<t<\gamma\left(x_{k}\right)^{2} .
\end{aligned}
$$

By proceeding in a similar way we obtain

$$
\left|K_{3}(x, y, t)\right| \leq C \gamma(y)^{-\delta} t^{-1+(\delta-n) / 2} e^{-c \frac{|x-y|^{2}}{t}}, x, y \in \mathbb{R}^{n} \text { and } 0<t<\gamma\left(x_{k}\right)^{2}
$$

Hence, since $\gamma(x) \sim \gamma(y) \sim \gamma\left(x_{k}\right)$ provided that $|x-y| \leq \gamma\left(x_{k}\right)$ and $x \in Q_{k}^{*}$, we conclude that $\left|\frac{\partial}{\partial t}\left(W_{t}^{\mathscr{L}}(x, y)-W_{t}(x, y)\right)\right| \leq C \gamma(y)^{-\delta} t^{-1+(\delta-n) / 2} e^{-c \frac{|x-y|^{2}}{t}}, x \in Q_{k}^{*},|x-y| \leq \gamma\left(x_{k}\right)$ and $0<t<\gamma\left(x_{k}\right)^{2}$

Therefore for some constants $C, c>0$ we get

$$
\begin{aligned}
\Omega_{5, k}(f)(x) & \leq C \int_{0}^{\gamma\left(x_{k}\right)^{2}} \frac{t^{\delta / 2-1}}{\gamma\left(x_{k}\right)^{\delta}} \int_{\mathbb{R}^{n}} \frac{e^{-c|x-y|^{2} / t}}{t^{n / 2}}|f(y)| d y d t \\
& \leq C \int_{0}^{\gamma\left(x_{k}\right)^{2}} \frac{t^{\delta / 2-1}}{\gamma\left(x_{k}\right)^{\delta}} \sum_{j=0}^{\infty} \frac{e^{-c 2^{2 j}}}{t^{n / 2}} \int_{|x-y| \leq 2^{j} \sqrt{t}}|f(y)| d y d t \\
& \leq C \int_{0}^{\gamma\left(x_{k}\right)^{2}} \frac{t^{\delta / 2-1}}{\gamma\left(x_{k}\right)^{\delta}} \sum_{j=0}^{\infty} \frac{2^{j n} e^{-c 2^{2 j}}}{\left(2^{j} \sqrt{t}\right)^{n}} \int_{|x-y| \leq 2^{j} \sqrt{t}}|f(y)| d y d t, \quad x \in Q_{k}^{*} .
\end{aligned}
$$


Moreover, by [13, Lemma 3.14], since $\gamma(x) \sim \gamma\left(x_{k}\right), x \in Q_{k}^{*}$,

$$
\begin{aligned}
\sum_{j=0}^{\infty} \frac{2^{j n} e^{-c 2^{2 j}}}{\left(2^{j} \sqrt{t}\right)^{n}} & \int_{|x-y| \leq 2^{j} \sqrt{t}}|f(y)| d y \\
\quad & \sum_{j \in \mathbb{N}, 2^{j} \sqrt{t} \leq \gamma(x)} \frac{2^{j n} e^{-c 2^{2 j}}}{\left(2^{j} \sqrt{t}\right)^{n}} \int_{|x-y| \leq 2^{j} \sqrt{t}}|f(y)| d y+\sum_{j \in \mathbb{N}, 2^{j} \sqrt{t}>\gamma(x)} \frac{2^{j n} e^{-c 2^{2 j}}}{\left(2^{j} \sqrt{t}\right)^{n}} \int_{|x-y| \leq 2^{j} \sqrt{t}}|f(y)| d y \\
& \leq C|| f \|_{\mathrm{BMO}^{\mathscr{L}}\left(\mathbb{R}^{n}\right)}\left(\sum_{j \in \mathbb{N}, 2^{j} \sqrt{t} \leq \gamma(x)} 2^{j n} e^{-c 2^{2 j}}\left(1+\log \frac{\gamma(x)}{2^{j} \sqrt{t}}\right)+\sum_{j \in \mathbb{N}, 2^{j} \sqrt{t}>\gamma(x)} 2^{j n} e^{-c 2^{2 j}}\right) \\
& \leq C\|f\|_{\mathrm{BMO}^{\mathscr{L}}\left(\mathbb{R}^{n}\right)}\left(\frac{\gamma\left(x_{k}\right)}{\sqrt{t}}\right)^{\varepsilon}, x \in Q_{k}^{*} \text { and } 0<t<\gamma\left(x_{k}\right)^{2},
\end{aligned}
$$

where $\varepsilon \in(0, \delta)$.

With this estimate we have that

$$
\Omega_{5, k}(f)(x) \leq C\|f\|_{\mathrm{BMO}^{\mathscr{L}}\left(\mathbb{R}^{n}\right)} \int_{0}^{\gamma\left(x_{k}\right)^{2}} \frac{t^{\delta / 2-1-\varepsilon / 2}}{\gamma\left(x_{k}\right)^{\delta-\varepsilon}} d t \leq C\|f\|_{\mathrm{BMO}^{\mathscr{L}}\left(\mathbb{R}^{n}\right)}, \quad x \in Q_{k}^{*} .
$$

Putting together (30) and (31), we infer $(A 2)$.

Comment: Se podria hacer notar que de hecho para A1 y A2 se probo algo mas fuerte, esto es, que la norma infinito del integrando estaba acotada por el miembro derecho ya que despues para la riesz adjunta lo necesitamos, ver pag. 17 de esta version

Next we notice that by (27) it follows that

$$
V_{\rho}\left(L_{k, t}\right)(f) \leq V_{\rho}\left(W_{t}^{\mathscr{L}}\right)(f)+V_{\rho}\left(H_{k, t}^{\mathscr{L}}\right)(f)+V_{\rho}\left(L_{k, t}^{\mathscr{L}}-L_{k, t}\right)(f) .
$$

By proceeding as in the proof of $\left(i_{k}\right)$ we get

$$
\int_{Q_{k}^{*}} V_{\rho}\left(W_{t}^{\mathscr{L}}\right)(f)(x) d x<\infty
$$

Then, $V_{\rho}\left(W_{t}^{\mathscr{L}}\right)(f)(x)<\infty$, a.e. $x \in Q_{k}^{*}$.

From $(30)$ and 31$)$ we deduce $V_{\rho}\left(L_{k, t}^{\mathscr{L}}-L_{k, t}\right)(f)(x)<\infty$, a.e. $x \in Q_{k}^{*}$. Also, by 28$)$ and $29 p, V_{\rho}\left(\underset{H_{k, t}}{\Phi}\right)(f)(x)<\infty$, a.e. $x \in Q_{k}^{*}$.

Hence, $V_{\rho}\left(L_{k, t}\right)(f)(x)<\infty$, a.e. $x \in Q_{k}^{*}$. We consider the following decomposition of $f$

$$
f=\left(f-f_{B}\right) \chi_{B^{*}}+\left(f-f_{B}\right) \chi_{\left(B^{*}\right)^{c}}+f_{B}=f_{1}+f_{2}+f_{3},
$$

where $B^{*}=2 B$.

Note that

$$
\begin{aligned}
V_{\rho}\left(L_{k, t}\right)\left(f_{1}\right)(x) & \leq C\left(\sup _{\left\{t_{j}\right\}_{j \in \mathbb{N}}, t_{j} \leq \gamma\left(x_{k}\right)^{2}}\left(\sum_{j=1}^{\infty}\left|W_{t_{j}}\left(f_{1}\right)(x)-W_{t_{j+1}}\left(f_{1}\right)(x)\right|^{\rho}\right)^{1 / \rho}\right. \\
& \left.+\sup _{0<t \leq \gamma\left(x_{k}\right)^{2}}\left|W_{t}\left(f_{1}\right)(x)\right|\right) \\
& \leq C\left(V_{\rho}\left(W_{t}\right)\left(f_{1}\right)(x)+W_{*}\left(f_{1}\right)(x)\right) .
\end{aligned}
$$

Then, since $W_{*}$ and $V_{\rho}\left(W_{t}\right)$ are bounded operators from $L^{2}\left(\mathbb{R}^{n}\right)$ into itself (see Theorem 2.1, we obtain

$$
\begin{aligned}
\int_{Q_{k}^{*}}\left|V_{\rho}\left(L_{k, t}\right)\left(f_{1}\right)(x)\right| d x & \leq C\left(\left|Q_{k}\right| \int_{B^{*}}\left|f(x)-f_{B}\right|^{2} d x\right)^{1 / 2} \\
& \leq C\left(|B|\left|Q_{k}\right|\right)^{1 / 2}\|f\|_{B M O^{\mathscr{E}}\left(\mathbb{R}^{n}\right)}<\infty .
\end{aligned}
$$

Hence $V_{\rho}\left(L_{k, t}\right)\left(f_{1}\right)(x)<\infty$, a.e. $x \in Q_{k}^{*}$.

Also, since $\int_{\mathbb{R}^{n}} W_{t}(x, y) d y=1, x \in \mathbb{R}^{n}$ and $t>0$, we get

$$
V_{\rho}\left(L_{k, t}\right)\left(f_{3}\right)=\left|f_{B}\right|<\infty, \quad x \in \mathbb{R}^{n} .
$$

Therefore, we deduce that $V_{\rho}\left(L_{k, t}\right)\left(f_{2}\right)(x)<\infty$, a.e. $x \in Q_{k}^{*}$.

Choosing $z_{1} \in B$ such that $V_{\rho}\left(L_{k, t}\right)\left(f_{2}\right)\left(z_{1}\right)<\infty$, we define $h_{B}(t)=L_{k, t}\left(f_{2}\right)\left(z_{1}\right), t \in(0, \infty)$. 
Suppose that $\left\{t_{j}\right\}_{j \in \mathbb{N}}$ is a real decreasing sequence that converges to zero and let $j_{k} \in \mathbb{N}$ be such that $t_{j_{k}} \leq \gamma\left(x_{k}\right)^{2}$ and $t_{j_{k}-1}>\gamma\left(x_{k}\right)^{2}$. We can write

$$
\begin{aligned}
& \left(\sum_{j=1}^{\infty}\left|L_{k, t_{j}}(f)(x)-L_{k, t_{j}}\left(f_{2}\right)\left(z_{1}\right)-\left(L_{k, t_{j+1}}(f)(x)-L_{k, t_{j+1}}\left(f_{2}\right)\left(z_{1}\right)\right)\right|^{\rho}\right)^{1 / \rho} \\
= & \left(\sum_{j=j_{k}}^{\infty}\left|W_{t_{j}}(f)(x)-W_{t_{j}}\left(f_{2}\right)\left(z_{1}\right)-\left(W_{t_{j+1}}(f)(x)-W_{t_{j+1}}\left(f_{2}\right)\left(z_{1}\right)\right)\right|^{\rho}+\left|W_{t_{j_{k}}}(f)(x)-W_{t_{j_{k}}}\left(f_{2}\right)\left(z_{1}\right)\right|^{\rho}\right)^{1 / \rho} \\
\leq & C\left(\left(\sum_{j=j_{k}}^{\infty}\left|W_{t_{j}}(f)(x)-W_{t_{j}}\left(f_{2}\right)\left(z_{1}\right)-\left(W_{t_{j+1}}(f)(x)-W_{t_{j+1}}\left(f_{2}\right)\left(z_{1}\right)\right)\right|^{\rho}\right)^{1 / \rho}\right. \\
+ & \sup _{0<t \leq \rho\left(x_{k}\right)^{2}}\left|W_{t}(f)(x)-W_{t}\left(f_{2}\right)\left(z_{1}\right)\right|
\end{aligned}
$$

and then

$$
\begin{aligned}
& \left|\left\|L_{k, t}(f)(x)\right\|_{E_{\rho}}-\left\|h_{B}\right\|_{E_{\rho}}\right| \\
\leq & \sup _{\left\{t_{j}\right\}_{j \in \mathbb{N} \downarrow} \downarrow, 0<t_{j} \leq \gamma\left(x_{k}\right)^{2}}\left(\sum_{j=1}^{\infty}\left|W_{t_{j}}(f)(x)-W_{t_{j}}\left(f_{2}\right)\left(z_{1}\right)-\left(W_{t_{j+1}}(f)(x)-W_{t_{j+1}}\left(f_{2}\right)\left(z_{1}\right)\right)\right|^{\rho}\right)^{1 / \rho} \\
+ & \sup _{0<t \leq \rho\left(x_{k}\right)^{2}}\left|W_{t}(f)(x)-W_{t}\left(f_{2}\right)\left(z_{1}\right)\right| .
\end{aligned}
$$

By taking into account Proposition 3.1 we obtain

Comment: tal vez convendria decir cual es la clase de sucesiones a las que se aplica la proposicion.

$$
\begin{aligned}
\frac{1}{|B|} \int_{B\left\{t_{j}\right\}_{j \in \mathbb{N} \downarrow} \downarrow, 0<t_{j} \leq \gamma\left(x_{k}\right)^{2}}\left(\sum_{j=1}^{\infty}\right. & \left.\left|W_{t_{j}}(f)(x)-W_{t_{j}}\left(f_{2}\right)\left(z_{1}\right)-\left(W_{t_{j+1}}(f)(x)-W_{t_{j+1}}\left(f_{2}\right)\left(z_{1}\right)\right)\right|^{\rho}\right)^{1 / \rho} d x \\
(32) & \leq C\|f\|_{B M O^{\mathscr{L}}\left(\mathbb{R}^{n}\right)} .
\end{aligned}
$$

Also, according to [13, Pages 348 and 349] it follows that

$$
\frac{1}{|B|} \int_{B} \sup _{0<t \leq \rho\left(x_{k}\right)^{2}}\left|W_{t}(f)(x)-W_{t}\left(f_{2}\right)\left(z_{1}\right)\right| d x \leq C\|f\|_{B M O^{\mathscr{L}}\left(\mathbb{R}^{n}\right)} .
$$

From $(32$ and 33 we deduce $(A 3)$.

Note that the constant $C>0$ does not depend on $k$ and $B$ in all the occurrences.

Thus the proof of $\left(i i_{k}\right)$ is finished.

4.2. Proof of Theorem 2.8, Let $\rho>2$. We may assume without loss of generality that $V \in R H_{q}$ with $q>n$. In fact, from ([17]), reverse Hölder classes are open, i.e., if $g \in R H_{s}$ it is also true that $g \in R H_{s+\varepsilon}$ for some $\varepsilon>0$.

In order to prove that the variation operator $V_{\rho}\left(R_{\ell}^{\mathscr{L}, \varepsilon}\right)$ is bounded from $B M O^{\mathscr{L}}\left(\mathbb{R}^{n}\right)$ into itself, we consider, for each $k \in \mathbb{N}$, the local operators defined as

$$
R_{\ell, k}^{\mathscr{L}}(f)(x)=P V \int_{|x-y|<\gamma\left(x_{k}\right)} R_{\ell}^{\mathscr{L}}(x, y) f(y) d y,
$$

and

$$
R_{\ell, k}(f)(x)=P V \int_{|x-y|<\gamma\left(x_{k}\right)} R_{\ell}(x-y) f(y) d y .
$$

Note that $\left|y-x_{k}\right| \leq 3 \gamma\left(x_{k}\right)$ when $x \in Q_{k}^{*}$ and $|x-y|<\gamma\left(x_{k}\right)$. Then, if $f \in \mathrm{BMO}^{\mathscr{L}}\left(\mathbb{R}^{n}\right)$,

$$
R_{\ell, k}(f)(x)=\lim _{\varepsilon \rightarrow 0^{+}} \int_{\varepsilon<|x-y|<\gamma\left(x_{k}\right)} R_{\ell}(x-y) f(y) \chi_{3 Q_{k}}(y) d y, \text { a.e. } x \in Q_{k}^{*},
$$

that is, this limit exists for almost all $x \in Q_{k}^{*}$ when $f \in \mathrm{BMO}^{\mathscr{L}}\left(\mathbb{R}^{n}\right)$. Also, $R_{\ell, k}^{\mathscr{L}}(f)(x)$ is defined for almost every $x \in Q_{k}^{*}$ when $f \in \mathrm{BMO}^{\mathscr{L}}\left(\mathbb{R}^{n}\right)$ (see [2, Proposition 1.1]). 
Let $f \in \mathrm{BMO}^{\mathscr{L}}\left(\mathbb{R}^{n}\right)$. We are going to analyze the properties $\left(i_{k}\right)$ and $\left(i i_{k}\right)$ when $H=$ $V_{\rho}\left(R_{\ell}^{\mathscr{L}, \varepsilon}\right)$. Let $k \in \mathbb{N}$. We can write

$$
\begin{aligned}
V_{\rho}\left(R_{\ell}^{\mathscr{L}, \varepsilon}\right)(f) & =\left(V_{\rho}\left(R_{\ell}^{\mathscr{L}, \varepsilon}\right)(f)-V_{\rho}\left(R_{\ell, k}^{\mathscr{L}, \varepsilon}\right)(f)\right) \\
& +\left(V_{\rho}\left(R_{\ell, k}^{\mathscr{L}, \varepsilon}\right)(f)-V_{\rho}\left(R_{\ell, k}^{\varepsilon}\right)(f)\right)+V_{\rho}\left(R_{\ell, k}^{\varepsilon}\right)(f) \\
& =F_{1, k}+F_{2, k}+V_{\rho}\left(R_{\ell, k}^{\varepsilon}\right)(f) .
\end{aligned}
$$

For $x \in Q_{k}^{*}$ we have

$$
\begin{aligned}
\left|F_{1, k}(x)\right| & \leq V_{\rho}\left(R_{\ell}^{\mathscr{L}, \varepsilon}-R_{\ell, k}^{\mathscr{L}, \varepsilon}\right)(f)(x) \\
& =\sup _{\left\{\varepsilon_{j}\right\}_{j \in \mathbb{N} \downarrow 0}}\left(\sum_{j=1}^{\infty}\left|\int_{\varepsilon_{j+1}<|x-y|<\varepsilon_{j}} R_{\ell}^{\mathscr{L}}(x, y) f(y) d y-\int_{\varepsilon_{j+1}<|x-y|<\varepsilon_{j},|x-y|<\gamma\left(x_{k}\right)} R_{\ell}^{\mathscr{L}}(x, y) f(y) d y\right|^{\rho}\right)^{1 / \rho} \\
& =\sup _{\left\{\varepsilon_{j}\right\}_{j \in \mathbb{N} \downarrow 0}}\left(\sum_{j=1}^{\infty}\left|\int_{\varepsilon_{j+1}<|x-y|<\varepsilon_{j},|x-y| \geq \gamma\left(x_{k}\right)} R_{\ell}^{\mathscr{L}}(x, y) f(y) d y\right|^{\rho}\right)^{1 / \rho} \\
& \leq \int_{|x-y|>\gamma\left(x_{k}\right)}\left|R_{\ell}^{\mathscr{L}}(x, y)\right||f(y)| d y, .
\end{aligned}
$$

Then, according to [4. Lemma 3, (a)], since $\gamma\left(x_{k}\right) \geq M \gamma(x), x \in Q_{k}^{*}$, for a certain $0<M<1$ that does not depend on $k \in \mathbb{N}$, it follows that

$$
\begin{aligned}
\left|F_{1, k}(x)\right| & \leq C \int_{|x-y|>M \gamma(x)} \frac{1}{|x-y|^{n}} \frac{1}{1+|x-y| / \gamma(x)}|f(y)| d y \\
& \leq C \sum_{j=0}^{\infty} \int_{M 2^{j} \gamma(x)<|x-y|<M 2^{j+1} \gamma(x)} \frac{1}{|x-y|^{n}} \frac{1}{1+|x-y| / \gamma(x)}|f(y)| d y \\
& \leq C \sum_{j=0}^{\infty} \frac{1}{2^{j}} \frac{1}{\left(2^{j} \gamma(x)\right)^{n}} \int_{|x-y|<2^{j+1} \gamma(x)}|f(y)| d y \leq C|| f \|_{\mathrm{BMO}^{\mathscr{L}}\left(\mathbb{R}^{n}\right)}, \quad x \in Q_{k}^{*} .
\end{aligned}
$$

Also, by using [4, Lemma 3, (b)], we obtain

$$
\begin{aligned}
\left|F_{2, k}(x)\right| & \leq V_{\rho}\left(R_{\ell, k}^{\mathscr{L}, \varepsilon}-R_{\ell, k}^{\varepsilon}\right)(f)(x) \\
& =\sup _{\left\{\varepsilon_{j}\right\}_{j \in \mathbb{N} \downarrow 0}}\left(\sum_{j=1}^{\infty}\left|\int_{\varepsilon_{j+1}<|x-y|<\varepsilon_{j},|x-y|<\gamma\left(x_{k}\right)}\left(R_{\ell}^{\mathscr{L}}(x, y)-R_{\ell}(x-y)\right) f(y) d y\right|^{\rho}\right)^{1 / \rho} \\
& \leq \int_{|x-y|<\gamma\left(x_{k}\right)}\left|R_{\ell}^{\mathscr{L}}(x, y)-R_{\ell}(x-y)\right||f(y)| d y \\
& \leq C \int_{|x-y|<\gamma\left(x_{k}\right)} \frac{1}{|x-y|^{n}}\left(\frac{|x-y|}{\gamma(x)}\right)^{2-n / q}|f(y)| d y, \quad x \in Q_{k}^{*} .
\end{aligned}
$$

Then, using Hölder inequality and that $\gamma(x) \sim \gamma\left(x_{k}\right)$ for $x \in Q_{k}^{*}$, we arrive to

$$
\begin{aligned}
\left|F_{2, k}(x)\right| & \leq C\left(\int_{|x-y|<\gamma\left(x_{k}\right)}|x-y|^{\left(2-n / q_{1}-n\right) r} d y\right)^{1 / r} \frac{1}{\gamma\left(x_{k}\right)^{2-n / q_{1}}}\left(\int_{|x-y|<\gamma\left(x_{k}\right)}|f(y)|^{r^{\prime}} d y\right)^{1 / r^{\prime}} \\
& \leq C\left(\frac{1}{\gamma\left(x_{k}\right)^{n}} \int_{|x-y|<\gamma\left(x_{k}\right)}|f(y)|^{r^{\prime}} d y\right)^{1 / r^{\prime}} \leq C\|f\|_{\mathrm{BMO}^{\mathscr{E}}\left(\mathbb{R}^{n}\right)}, \quad x \in Q_{k}^{*} .
\end{aligned}
$$

Here, $1<r<n /(n-2+n / q)$.

Since, for $i=1,2, F_{i, k} \in L^{\infty}\left(Q_{k}^{*}\right)$ and $\left\|F_{i, k}\right\|_{L^{\infty}\left(Q_{k}^{*}\right)} \leq C\|f\|_{\mathrm{BMO}^{\mathscr{L}}\left(\mathbb{R}^{n}\right)}$, where $C$ does not depend on $k \in \mathbb{N}$, in order to see that the properties $\left(i_{k}\right)$ and $\left(i i_{k}\right)$ hold for $H=V_{\rho}\left(R_{\ell}^{\mathscr{L}, \varepsilon}\right)$ it is sufficient to establish those properties for $H=V_{\rho}\left(R_{\ell, k}^{\varepsilon}\right)$. 
Fix again $k \in \mathbb{N}$. Then

$$
\begin{aligned}
V_{\rho}\left(R_{\ell, k}^{\varepsilon}\right)(f)(x) & =\sup _{\left\{\varepsilon_{j}\right\}_{j \in \mathbb{N} \downarrow 0}}\left(\sum_{j=1}^{\infty}\left|\int_{\varepsilon_{j+1}<|x-y|<\varepsilon_{j},|x-y|<\gamma\left(x_{k}\right)} R_{\ell}(x-y) f(y) d y\right|^{\rho}\right)^{1 / \rho} \\
& =\sup _{\left\{\varepsilon_{j}\right\}_{j \in \mathbb{N} \downarrow 0}}\left(\sum_{j=1}^{\infty}\left|\int_{\varepsilon_{j+1}<|x-y|<\varepsilon_{j},|x-y|<\gamma\left(x_{k}\right)} R_{\ell}(x-y) f(y) \chi_{Q_{k}^{* *}}(y) d y\right|^{\rho}\right)^{1 / \rho} \\
& \leq V_{\rho}\left(R_{\ell, k}^{\varepsilon}\right)\left(f \chi_{Q_{k}^{* *}}\right)(x), \quad x \in Q_{k}^{*} .
\end{aligned}
$$

Hence, according to Theorem 2.7, (i), we have

$$
\begin{aligned}
\frac{1}{\left|Q_{k}\right|} \int_{Q_{k}}\left|V_{\rho}\left(R_{\ell, k}^{\varepsilon}\right)(f)(x)\right| d x & \leq\left(\frac{1}{\left|Q_{k}\right|} \int_{Q_{k}}\left|V_{\rho}\left(R_{\ell, k}^{\varepsilon}\right)\left(f \chi_{Q_{k}^{* *}}\right)(x)\right|^{2} d x\right)^{1 / 2} \\
& \leq C\left(\frac{1}{\left|Q_{k}\right|} \int_{Q_{k}^{* *}}|f(x)|^{2} d x\right)^{1 / 2} \leq C\|f\|_{\mathrm{BMO}^{\mathscr{L}}\left(\mathbb{R}^{n}\right)} .
\end{aligned}
$$

Let now $x_{0} \in \mathbb{R}^{n}$ and $r_{0}>0$ such that $B=B\left(x_{0}, r_{0}\right) \subset Q_{k}^{*}$. Then, by using Proposition 3.2 we can see

$$
\frac{1}{|B|} \int_{B}\left|V_{\rho}\left(R_{\ell, k}^{\varepsilon}\right)(f)(x)-V_{\rho}\left(R_{\ell, k}^{\varepsilon}\right)\left(f_{2}\right)\left(z_{1}\right)\right| d x \leq C|| f \|_{\mathrm{BMO}^{\mathscr{L}}\left(\mathbb{R}^{n}\right)} .
$$

where $f_{2}=\left(f-f_{B}\right) \chi_{\left(B^{* *}\right)^{c}}$ and $z_{1} \in B$ is such that $V_{\rho}\left(R_{\ell, k}^{\varepsilon}\right)\left(f_{2}\right)\left(z_{1}\right)<\infty$. Hence, $V_{\rho}\left(R_{\ell, k}^{\varepsilon}\right)(f) \in$ $\operatorname{BMO}\left(Q_{k}^{*}\right)$ and

$$
\left\|V_{\rho}\left(R_{\ell, k}^{\varepsilon}\right)(f)\right\|_{\mathrm{BMO}\left(Q_{k}^{*}\right)} \leq C\|f\|_{\mathrm{BMO}^{\mathscr{L}}\left(\mathbb{R}^{n}\right)} .
$$

Note that the constants $C>0$ appearing in (34) and (35) do not depend on $k \in \mathbb{N}$. Thus the proof of the desired result is finished.

Assume now that $V \in R H_{q}$ with $n / 2<q$. In fact it is sufficient to consider $n / 2<q<n$. We have to show that the variation operator for the adjoint Riesz transform, $V_{\rho}\left(\mathscr{R}_{\ell}^{\mathscr{L}, \varepsilon}\right)$, is bounded from $B M O^{\mathscr{L}}\left(\mathbb{R}^{n}\right)$ into itself. Looking at the proof above, we notice that the properties on $\mathscr{R}_{\ell}^{\mathscr{L}}$ needed to make the above proof work are, on one side, its boundedness on $L^{2}\left(\mathbb{R}^{n}\right)$, which is true by ([29, Theorem 0.5 and 0.8$]$ ), and, on the other side that the operators defined by

and

$$
T_{1, k}(f)(x)=\int_{|x-y|>\gamma\left(x_{k}\right)}\left|R_{\ell}^{\mathscr{L}}(y, x)\right||f(y)| d y,
$$

$$
T_{2, k}(f)(x)=\int_{|x-y|<\gamma\left(x_{k}\right)}\left|R_{\ell}^{\mathscr{L}}(y, x)-R_{\ell}(y-x)\right||f(y)| d y,
$$

map $\mathrm{BMO}^{\mathscr{L}}\left(\mathbb{R}^{n}\right)$ into $L^{\infty}\left(Q_{k}^{*}\right)$, and that for $i=1,2$,

$$
\left\|T_{i, k}(f)\right\|_{L^{\infty}\left(Q_{k}^{*}\right)} \leq C\|f\|_{\mathrm{BMO}^{\mathscr{L}}\left(\mathbb{R}^{n}\right)},
$$

where $C>0$ does not depend on $k \in \mathbb{N}$.

Let $k \in \mathbb{N}$ and $f \in \mathrm{BMO}^{\mathscr{L}}\left(\mathbb{R}^{n}\right)$. According to [29, p. 538], we have that

$$
\begin{aligned}
\left|T_{1, k}(f)(x)\right| \leq & C\left(\int_{|x-y|>\gamma\left(x_{k}\right)} \frac{1}{|x-y|^{n}} \frac{1}{(1+|x-y| / \gamma(x))^{\alpha}}|f(y)| d y\right. \\
& \left.+\int_{|x-y|>\gamma\left(x_{k}\right)} \frac{1}{|x-y|^{n-1}} \frac{|f(y)|}{(1+|x-y| / \gamma(x))^{\alpha}} \int_{|y-z|<\frac{|x-y|}{4}} \frac{V(z)}{|z-y|^{n-1}} d z d y\right) \\
= & C\left(T_{1,1, k}(f)(x)+T_{1,2, k}(f)(x)\right), \quad x \in Q_{k}^{*},
\end{aligned}
$$

where $\alpha>0$ will be chosen later large enough.

As it was shown earlier, we have

$$
\left\|T_{1,1, k}(f)\right\|_{L^{\infty}\left(Q_{k}^{*}\right)} \leq C\|f\|_{\mathrm{BMO}^{\mathscr{L}}\left(\mathbb{R}^{n}\right)},
$$

provided that $\alpha \geq 1$. 
On the other hand, since $\gamma(x) \sim \gamma\left(x_{k}\right)$ when $x \in Q_{k}^{*}$, we can write

$$
\begin{aligned}
\left|T_{1,2, k}(f)(x)\right| \leq & C \sum_{j=0}^{\infty} \frac{1}{2^{j \alpha}\left(2^{j} \gamma\left(x_{k}\right)\right)^{n-1}} \int_{2^{j} \gamma\left(x_{k}\right)<|x-y| \leq 2^{j+1} \gamma\left(x_{k}\right)}|f(y)| \int_{B\left(y, \frac{|x-y|}{4}\right)} \frac{V(z)}{|z-y|^{n-1}} d z d y \\
\leq & C \sum_{j=0}^{\infty} \frac{1}{2^{j \alpha}\left(2^{j} \gamma\left(x_{k}\right)\right)^{n-1}}\left(\int_{|x-y| \leq 2^{j+1} \gamma\left(x_{k}\right)}|f(y)|^{q_{0}^{\prime}} d y\right)^{1 / q_{0}^{\prime}} \\
& \times\left(\int_{\mathbb{R}^{n}}\left|\int_{|x-z|<2^{j+2} \gamma\left(x_{k}\right)} \frac{V(z)}{|z-y|^{n-1}} d z\right|^{q_{0}} d y\right)^{1 / q_{0}}, \quad x \in Q_{k}^{*},
\end{aligned}
$$

where $\frac{1}{q_{0}}=\frac{1}{q}-\frac{1}{n}$. Then, the $L^{p}$-boundedness properties of the classical fractional integrals (31]) lead us to

$\left|T_{1,2, k}(f)(x)\right| \leq C|| f \|_{\mathrm{BMO}^{\mathscr{L}}\left(\mathbb{R}^{n}\right)} \sum_{j=0}^{\infty} \frac{1}{2^{j \alpha}\left(2^{j} \gamma\left(x_{k}\right)\right)^{n-1-n / q_{0}^{\prime}}}\left(\int_{|x-z|<2^{j+2} \gamma\left(x_{k}\right)}|V(z)|^{q} d z\right)^{1 / q}, x \in Q_{k}^{*}$.

By using the properties of $V$ and $\gamma$ (4, Lemma 1]) we obtain, for a certain $\mu>0$,

$$
\left(\int_{|x-z|<2^{j+2} \gamma\left(x_{k}\right)}|V(z)|^{q} d z\right)^{1 / q} \leq C\left(2^{j} \gamma\left(x_{k}\right)\right)^{-n / q^{\prime}} 2^{j \mu} \gamma\left(x_{k}\right)^{n-2}, \quad x \in Q_{k}^{*} .
$$

By choosing $\alpha>0$ large enough, it follows that

$$
\begin{aligned}
\left|T_{1,2, k}(f)(x)\right| & \leq C\|f\|_{\mathrm{BMO}^{\mathscr{L}}\left(\mathbb{R}^{n}\right)} \sum_{j=0}^{\infty} \frac{1}{2^{j\left(\alpha+n / q_{0}-1+n / q^{\prime}-\mu\right)}} \\
& \leq C\|f\|_{\mathrm{BMO}^{\mathscr{E}}\left(\mathbb{R}^{n}\right)}, \quad x \in Q_{k}^{*} .
\end{aligned}
$$

We conclude from (36) and (37) that

$$
\left\|T_{1, k}(f)\right\|_{L^{\infty}\left(Q_{k}^{*}\right)} \leq C\|f\|_{\mathrm{BMO}^{\mathscr{L}}\left(\mathbb{R}^{n}\right)},
$$

where $C>0$ does not depend on $k \in \mathbb{N}$.

According to [29, (5.9)] we get

$$
\begin{aligned}
\left|T_{2, k}(f)(x)\right| & \leq C\left(\int_{|x-y|<\gamma\left(x_{k}\right)} \frac{1}{|x-y|^{n}}\left(\frac{|x-y|}{\gamma(x)}\right)^{2-n / q}|f(y)| d y\right. \\
& \left.+\int_{|x-y|<\gamma\left(x_{k}\right)} \frac{1}{|x-y|^{n-1}} \int_{|y-z|<\frac{|x-y|}{4}} \frac{V(z)}{|z-y|^{n-1}} d z|f(y)| d y\right) \\
& =C\left(T_{2,1, k}(f)(x)+T_{2,2, k}(f)(x)\right), x \in Q_{k}^{*} .
\end{aligned}
$$

As in the proof in the first part of this theorem we have that

$$
\left\|T_{2,1, k}(f)\right\|_{L^{\infty}\left(Q_{k}^{*}\right)} \leq C\|f\|_{\mathrm{BMO}^{\mathscr{L}}\left(\mathbb{R}^{n}\right)} .
$$

Also, we can write

$$
\begin{aligned}
& \left|T_{2,2, k}(f)(x)\right| \leq C \sum_{j=0}^{\infty} \int_{2^{-j-1} \gamma\left(x_{k}\right) \leq|x-y|<2^{-j} \gamma\left(x_{k}\right)} \frac{|f(y)|}{\left(2^{-j} \gamma\left(x_{k}\right)\right)^{n-1}} \int_{|x-z|<2^{-j+1} \gamma\left(x_{k}\right)} \frac{V(z)}{|y-z|^{n-1}} d z d y \\
& \leq C \sum_{j=0}^{\infty} \frac{1}{\left(2^{-j} \gamma\left(x_{k}\right)\right)^{n-1}}\left(\int_{|x-y|<2^{-j} \gamma\left(x_{k}\right)}|f(y)|^{q_{0}^{\prime}} d y\right)^{1 / q_{0}^{\prime}} \\
& \times\left(\int_{\mathbb{R}^{n}}\left(\int_{|x-z|<2^{-j+1} \gamma\left(x_{k}\right)} \frac{V(z)}{|y-z|^{n-1}} d z\right)^{q_{0}} d y\right)^{1 / q_{0}} \\
& \leq C \sum_{j=0}^{\infty} \frac{1}{\left(2^{-j} \gamma\left(x_{k}\right)\right)^{n-1-n / q_{0}^{\prime}}}\left(\int_{|x-z|<2^{-j+1} \gamma\left(x_{k}\right)} V(z)^{q} d z\right)^{1 / q} \\
& \times\left(\frac{1}{\left(2^{-j} \gamma\left(x_{k}\right)\right)^{n}} \int_{|x-y|<2^{-j} \gamma\left(x_{k}\right)}|f(y)|^{q_{0}^{\prime}} d y\right)^{1 / q_{0}^{\prime}}, \quad x \in Q_{k}^{*},
\end{aligned}
$$


where $\frac{1}{q_{0}}=\frac{1}{q}-\frac{1}{n}$.

Since $V \in R H_{q}$ and $\gamma(x) \sim \gamma\left(x_{k}\right)$, when $x \in Q_{k}^{*}$, we have ([4, Lemma 1])

$$
\left(\int_{B\left(x, 2^{-j+1} \gamma\left(x_{k}\right)\right)} V(z)^{q} d z\right)^{1 / q} \leq C \gamma\left(x_{k}\right)^{n / q-2}, \quad x \in Q_{k}^{*} .
$$

Moreover, an argument like the one used to show [13, Lemma 2] allows us to get

$$
\left(\frac{1}{\left(2^{-j} \gamma\left(x_{k}\right)\right)^{n}} \int_{|x-y|<2^{-j} \gamma\left(x_{k}\right)}|f(y)|^{q_{0}^{\prime}} d y\right)^{1 / q_{0}^{\prime}} \leq C j\|f\|_{\mathrm{BMO}^{\mathscr{L}}\left(\mathbb{R}^{n}\right)} .
$$

Then,

$$
\begin{aligned}
\left|T_{2,2, k}(f)(x)\right| & \leq C \sum_{j=0}^{\infty} \frac{j}{\left(2^{-j} \gamma\left(x_{k}\right)\right)^{n / q_{0}-1}} \gamma\left(x_{k}\right)^{n / q-2}\|f\|_{\mathrm{BMO}^{\mathscr{E}}\left(\mathbb{R}^{n}\right)} \\
& \leq C\|f\|_{\mathrm{BMO}^{\mathscr{L}}\left(\mathbb{R}^{n}\right)}, \quad x \in Q_{k}^{*} .
\end{aligned}
$$

Note that $\frac{n}{q_{0}}-1=\frac{n}{q}-2<0$.

By combining (38) and $(39)$ we conclude that

$$
\left\|T_{2, k}(f)\right\|_{L^{\infty}\left(Q_{k}^{*}\right)} \leq C\|f\|_{\mathrm{BMO}^{\mathscr{L}}\left(\mathbb{R}^{n}\right)},
$$

where $C>0$ does not depend on $k \in \mathbb{N}$.

Thus the proof is finished.

\section{REFERENCES}

[1] C. Bennet, R.A. DeVore, and R. Sharpley, Weak- $L^{\infty}$ and BMO, Ann. of Math. (2) 113 (3) (1981), 601-611.

[2] J.J. Betancor, J.C. Fariña, E. Harboure and L. Rodríguez-Mesa, $L^{p}$-boundedness properties of variation and oscillation operators in the Schródinger setting, preprint.

[3] B. Bongioanni, E. Harboure, and O. Salinas, Weighted inequalities for negative powers of Schrödinger operators, J. Math. Anal. Appl. 348 (2008), no. 1, 12-27.

[4] _ Riesz transform related to Schrödinger operators acting on BMO type spaces, J. Math. Anal. Appl. 357 (2009), 115-131.

[5] — Commutators of Riesz transform related to schrödinger operators, J. Fourier Anal. Appl., (DOI:10.1007/S00041-010-9133-6).

[6] J. Bourgain, Pointwise ergodic theorems for arithmetic sets, Publ. Math., IHES 69 (1989), 5-45.

[7] J.T. Campbell, R.L. Jones, K. Reinhold, and M. Wierdl, Oscillation and variation for the Hilbert transform, Duke Math. J. 105 (2000), no. 1, 59-83.

[8] _ Oscillation and variation for singular integrals in higher dimensions, Trans. Amer. Math. Soc. 355 (2003), no. 5, 2115-2137.

[9] R. R. Coifman, R. Rochberg, and G. Weiss, Factorization theorems for Hardy spaces in several variables, Ann. of Math. (2) 103 (1976), no. 3, 611-635.

[10] R. Crescimbeni, F. Martín-Reyes, A. de la Torre, and J.L. Torrea, The $\rho$-variation of the Hermitian Riesz transform, Acta Math. Sin. (Engl. Ser.) (to appear).

[11] R. Crescimbeni, R. A. Macías, T. Menárguez, J. L. Torrea, and B. Viviani, The $\rho$-variation as an operator between maximal operators and singular integrals, J. Evol. Equ. 9 (2009), no. 1, 81-102.

[12] J.-F. Dong and H.-P. Liu, The BMOL space and Riesz transforms associated with Schrödinger operators, Acta Math. Sin., 26 (4) (2010), 659-668.

[13] J. Dziubański, G. Garrigós, T. Martínez, J. L. Torrea, and J. Zienkiewicz, BMO spaces related to Schrödinger operators with potentials satisfying a reverse Hölder inequality, Math. Z. 249 (2005), no. 2, 329-356.

[14] J. Dziubański, and J. Zienkiewicz, Hardy space $H^{1}$ associated to Schrödinger operator with potential satisfying reverse Hölder inequality, Revista Mat. Iberoamericana 15 (2) (1999), 279-296. 
[15] J. Dziubański, and J. Zienkiewicz, $H^{p}$ spaces for Schrödinger operators, Fourier Analysis and Related Topics, Vol. 56, Banach Center Publications, 2002, 45-53.

[16] J. Dziubański, and J. Zienkiewicz, $H^{p}$ spaces associated with Schrödinger operators with potentials from reverse Hölder classes, Colloq. Math. 98 (1) (2003), 5-38.

[17] F. W. Gehring, The $L^{p}$-integrability of the partial derivatives of a quasiconformal mapping, Acta Math. 130 (1973), 265-277.

[18] T. A. Gillespie and J. L. Torrea, Dimension free estimates for the oscillation of Riesz transforms, Israel J. Math. 141 (2004), 125-144. MR MR2063029 (2005e:47085)

[19] Z. Guo, P. Li, and L. Peng, L $L^{p}$ boundedness of commutators of Riesz transforms associated to Schrödinger operator, J. Math. Anal. Appl. 341 (2008), 421-432.

[20] E. Harboure, R. Macías, T. Menárguez, and J.L. Torrea, Oscillation and variation for the Gaussian Riesz transforms and Poisson integral, Proc. Roy. Soc. Edinburgh Sect. A 135 (1) (2005) 85-104.

[21] R.L. Jones, R. Kaufman, J. Rosenblatt and M. Wierdl, Oscillation in ergodic theory, Ergodic. Th. and Dynam. Sys. 18 (4) (1998), 889-936.

[22] R. L. Jones and K. Reinhold, Oscillation and variation inequalities for convolution powers, Ergodic Theory Dynam. Systems 21 (2001), no. 6, 1809-1829.

[23] R.L. Jones, A. Seeger and J. Wright, Strong variational and jump inequalities in harmonic analysis, Trans. Amer. Math. Soc. 360 (12) (2008), 6711-6742.

[24] R.L. Jones and G. Wang, Variation inequalities for the Fejer and Poisson kernels, Trans. Amer. Math. Soc. 356 (11) (2004), 4493-4518.

[25] Y. Liu and Y. Ding, Some estimates of Schrödinger-type operators with certain nonnegative potentials, Internat. J. Math. Math. Sci., 2008, Art. ID 214030, 8 pp. .

[26] Y. Liu and J. Dong, Some estimates of higher order Riesz transform related to Schrödinger-type operators, Potential Analysis 32 (1) (2010), 41-55.

[27] R. Oberlin, A. Seeger. T. Tao, C. Thiele, and J. Wright, A variation norm Carleson theorem, Arxiv:0910.1555.

[28] Z. W. Shen, On the Neumann problem for Schrödinger operators in Lipschitz domains, Indiana Univ. Math. J. 43 (1) (1994), 143-176.

[29] Z. W. Shen, $L^{p}$ estimates for Schrödinger operators with certain potentials, Ann. Inst. Fourier (Grenoble) 45 (1995), no. 2, 513-546.

[30] E. M. Stein, Topics in harmonic analysis related to the Littlewood-Paley theory., Annals of Mathematics Studies, No. 63, Princeton University Press, Princeton, N.J., 1970.

[31] _ Harmonic analysis: real-variable methods, orthogonality, and oscillatory integrals, 43 (1993), xiv +695 , With the assistance of Timothy S. Murphy, Monographs in Harmonic Analysis, III.

[32] S. Sugano, $L^{p}$ estimates for some Schrödinger operators and a Calderón-Zygmund operator of Schrödinger type, Tokyo J. Math. 30 (2007), 179-197.

[33] L. Tang, and J. Dong, Boundedness for some Schrödinger type operators on Morrey spaces related to certain nonnegative potentials, J. Math. Anal. Appl. 355 (2009), 101-109.

[34] A. Torchinsky, Real-variable methods in harmonic analysis, Dover Publications, Inc., Mineola, New York, 2004.

[35] Dachun Yang, Dongyong Yang and Y. Zhou, Endpoint properties of localized Riesz transforms and fractional integrals associated to Schrödinger operators, Potential Analysis 30 (2009), 271-300.

[36] J. Zhong, Harmonic analysis for some Schrödinger type operators, Ph. D. Thesis, Princeton University, 1993. 
Departamento de Análisis Matemático, Universidad de la Laguna, Campus de Anchieta, Avda. Astrofísico Francisco Sánchez, s/n, 38271 La Laguna (Sta. Cruz de Tenerife), Spain

E-mail address: jbetanco@ull.es

Departamento de Análisis Matemático, Universidad de la Laguna, Campus de Anchieta, Avda. Astrofísico Francisco Sánchez, s/n, 38271 La Laguna (Sta. Cruz de Tenerife), Spain

E-mail address: jcfarina@ull.es

Instituto de Matemática Aplicada del Litoral, imal, Universidad Nacional del Litoral, C/ Gëmes, Santa Fe, Argentina

Departamento de Análisis Matemático, Universidad de la laguna, Campus de Anchieta, Avda. Astrofísico Francisco Sánchez, s/n, 38271 La Laguna (Sta. Cruz de Tenerife), Spain

E-mail address: jrguez@ull.es 